\title{
Valuing Equity-Linked Death Benefits in a Regime-Switching Framework
}

\author{
Chi Chung Siu ${ }^{\dagger}$, Sheung Chi Phillip Yam ${ }^{\ddagger}$, and Hailiang Yang* \\ ${ }^{\dagger}$ Finance Discipline Group, Business School, University of Technology, Sydney \\ Sydney, NSW, Australia. \\ ${ }^{\ddagger}$ Department of Statistics, The Chinese University of Hong Kong, \\ Shatin, N.T., Hong Kong. \\ * Department of Statistics and Actuarial Science, The University of Hong Kong, \\ Pokfulam Road, Hong Kong.
}

\begin{abstract}
In this article, we consider the problem of computing the expected discounted value of a death benefit, e.g. in Gerber et al. (2012, 2013), in a regime-switching economy. Contrary to their proposed discounted density approach, we adopt the Laplace transform to value the contingent options. By this alternative approach, closed-form expressions for the Laplace transforms of the values of various contingent options, such as call/put options, lookback options, barrier options, dynamic fund protection, and the dynamic withdrawal benefits, have been obtained. The value of each contingent option can then be recovered by the numerical Laplace inversion algorithm, and this efficient approach is documented by several numerical illustrations. The strength of our methodology becomes apparent when we tackle the valuations of exotic contingent options in the cases when (1) the contracts have a finite expiry date; (2) when the time-until-death variable is uniformly distributed in accordance with De Moivre's law.
\end{abstract}

Keywords: Regime switching; Jump-diffusion process; Equity-linked death benefits; Guaranteed minimum death benefits; First passage probabilities.

\section{Introduction}

To accommodate clients with different preferences, many life insurance companies offer insurance protections embedded with different features such as interest rate guarantees, bonus and surrender options, participating policies, and equity-linked policies. The fair values for these option-embedded life insurance contracts are crucial to insurance companies for financial reporting purposes.

Since the late 1970's, option pricing theory has been applied to the fair pricing of these life insurance contracts. To see this, let us consider the guaranteed minimum death benefit 
(hereafter, GMDB) in a variable annuity for an individual age $\mathrm{x}$ with the guaranteed payment in case of his death,

$$
\max \left(S_{T_{\mathrm{x}}}, K\right)=\underbrace{S_{T_{\mathrm{x}}}}_{\text {Account value }}+\underbrace{\left(K-S_{T_{\mathrm{x}}}\right)^{+}}_{\text {Put option payoff }}
$$

where $T_{\mathrm{x}}$ is the time-until-death random variable and $K$ is the guaranteed minimum amount. The second term on the right-hand side of (1) constitutes the put option with strike price $K$ and maturity $T_{\mathrm{x}} \cdot{ }^{1}$ Early works focused on the valuation of life insurance products when $\left(S_{t}\right)_{t \geq 0}$ follows a geometric Brownian motion. Brennan and Schwartz $(1976,1979)$ employed the Black-Scholes formula to value life insurance products with different options. Boyle and Schwartz (1977) investigated different dimensions of equity-linked life insurance policies in addition to those in Brennan Schwartz (1976). Ronn and Verma (1986) provided an empirical estimate for deposit insurance premiums. Albizzati and Geman (1994) provided the fair value of surrender options in the life insurance policies. Bacinello (2001) studied the fair valuation of participating policies with minimum rate guarantees. Milevsky and Posner (2001) evaluated fair insurance risk fees for GMDBs in variable annuities (VAs) and some mutual funds. Lee (2003) proposed another type of equity-indexed annuity embedded with path-dependent options that can increase the participation rates.

As discussed in Gerber et al. (2013) and the references therein, modeling the stock price process as a jump-diffusion process, i.e. Brownian motion plus an independent Poisson process, provides important economic features for its ability in capturing the empirical facts such as volatility smiles and heavy tails, as, for example, documented in Merton (1976) that are absent in the Brownian motion framework. Among the popular jump-diffusion models, it is also well-recognized that the jump-diffusion models become highly tractable when the distribution of the jump sizes can be represented as a combination of exponential distributions. Examples include Dufresne and Gerber (1988, 1991), Chan (1990), Gerber et al. (2006), and Dong (2011) on the closed-form expressions for the probability of ruin, discounted dividends until ruin, and the applications in the fair valuations of the option-embedded insurance contracts under this special jump-diffusion model.

As the durations of life insurance contracts amount to years or even decades, these contracts should subject to the changes of economic regimes. Yet, the aforementioned literature focused on the valuations of option-embedded insurance contracts under models with constant or time-dependent coefficients. In this respect, Markov regime-switching models, in which the dynamics of the change of economic regimes is modeled by a Markov chain, capture the state-dependent effect while maintaining a high degree of tractability. One important work on Markov switching models is Hamilton (1989), in which he introduced a tractable dynamic model with Markov regime-switching between regimes to capture the endogenous structural breaks in the business cycle. Moreover, Hardy (2001) showed that the regime-switching lognormal model provides significant improvement over all other models in the sense of maximizing the likelihood function when she fitted different models on the monthly data from the Standard \& Poor's 500 and the Toronto Stock Exchange 300 indices. For the fair valuations

\footnotetext{
${ }^{1}$ Equivalently, the same GMDB in a variable annuity can also be expressed in terms of the call option by the following relation:

$$
\max \left(S_{T_{\mathrm{x}}}, K\right)=\underbrace{K}_{\text {Account value }}+\underbrace{\left(S_{T_{\mathrm{x}}}-K\right)^{+}}_{\text {Call option payoff }} .
$$
}


of the financial and insurance products under the Markov regime-switching models, see, for example, Siu (2005), Boyle and Draviam (2007), Elliott and Siu (2009), and Yuen and Yang (2009).

In this article, we tackle the valuation of variable annuities via the Laplace transform instead of the density approach proposed in Gerber et al. (2012, 2013), as the density of the regimeswitching jump-diffusion process lacks a tractable form. We first assume that the time-untildeath random variable $T_{\mathrm{x}}$ of a person at age $\mathrm{x}$ is represented as a combination of exponential distributions, independent of the underlying asset dynamics. Representing the distribution of $T_{\mathrm{x}}$ as a combination of the exponential distributions has two advantages. First, it will become clear in the paper that the valuation of the variable annuities under the exponentiallydistributed $T_{\mathrm{x}}$ is equivalent to the Laplace transform of the contingent claims with respect to the maturity T. Moreover, we show that the variable annuities in Gerber et al. $(2012,2013)$ retain closed-form expressions to a large extent, when we also assume that the underlying asset dynamics exhibit exponentially-distributed jump sizes in each regime. The tractability is made possible due to the fact that we can exploit the elementary expression for the first passage time distribution developed in Siu (2012), thereby facilitating us to compute the fair values of GMDBs under which distribution of the maximum or minimum values of the assets is required. Secondly, the exponentially-distributed $T_{\mathrm{x}}$ is not a strong modeling restriction due to the fundamental result that all combinations of exponential distributions are weakly dense in the space of all probability distributions on the positive axis (see Botta and Harris (1986)). In light of this, we expand the scope of the Laplace transform framework by valuing GDMBs using the combination of exponential distributions calibrated to a life table.

The rest of the paper is organized as follows. Section 2 introduces the model settings. Section 3 presents analytical valuations of various GMDBs. In Section 4, we investigate the valuation problem of $T$-year options and the case of a uniformly-distributed mortality density function. Section 5 provides numerical illustrations for the cases when $T_{\mathrm{x}}$ is exponentially distributed and when mortality rates are obtained from the life table. Section 6 concludes the paper. Appendix A provides the derivation of the first passage time distribution of the model process. As will become clear in the paper, valuing fractional lookback options under the regime-switching framework requires additional model assumptions. For the sake of completeness, we provide discussion of valuing fractional lookback option in Appendix B. Appendix $\mathrm{C}$ reviews the numerical Laplace inversion algorithm employed in the paper.

\section{Model Formulation}

Define a probability space by the usual triplet $(\Omega, \mathcal{F}, \mathbb{Q})$, where $\mathcal{F}=\left(\mathcal{F}_{t}\right)_{t \geq 0}$ denotes the filtration generated by the random processes described below and $\mathbb{Q}$ denotes the risk-neutral measure under which every discounted price process is a martingale. ${ }^{2}$

\footnotetext{
${ }^{2}$ Since insurance products are not traded in the market, and that the model in this paper lies in the realm of incomplete market, there exist infinitely many equivalent martingale measures. As in Dong (2011), we hereafter assume that the equivalent martingale measure $\mathbb{Q}$ under which that every discounted price process is a $\mathbb{Q}$-martingale has been chosen and call this chosen measure a risk-neutral measure. See, for example, Schweizer (2010) and the references therein for the optimal selection of risk-neutral measure in the context of minimal entropy martingale measure under the incomplete market setting.
} 


\subsection{Asset price dynamics $S_{t}$}

Following Elliott et al. (1994), we model the evolution of the state of the economy over time via a Markov chain.

Let $\left(J_{t}\right)_{t \geq 0}$ be a continuous-time, time-homogeneous, Markov chain on a finite state space $E=\{1, \ldots, d\}$. More precisely, $\left(J_{t}\right)_{t \geq 0}$ is right-continuous and irreducible on $E$ such that $J_{t}=i$ means the state of the economy at time $t$ is $i$.

Let $\mathbf{Q}$ be the intensity matrix of $J_{t}$ with respect to the Lebesgue measure, i.e.

$$
\mathbf{Q}=\left\{q_{i j}\right\}_{i, j \in E},
$$

where $q_{i j} \geq 0$, for $i \neq j, \quad q_{i i}=-\sum_{i \neq j} q_{i j} \leq 0$.

Let $\left\{\left(X_{t}^{j}\right)_{t \geq 0} ; j \in E\right\}$ be a family of mutually independent, double-exponential jump diffusion processes (see Kou (2002)), i.e.

$$
X_{t}^{j}=X_{0}^{j}+b_{j} t+\sigma_{j} W_{t}^{j}+N_{t}^{j}
$$

where $X_{0}^{j} \in \mathbb{R}$ is a constant and $\left(W_{t}^{j}\right)_{t \geq 0}$ denotes a standard Brownian motion; $b_{j}$ and $\sigma_{j}>0$ are constants describing the drift and volatility of $X^{j}$, respectively; and $\left(N_{t}^{j}\right)_{t \geq 0}$ is a compound Poisson process, independent of $\left(W_{t}^{j}\right)_{t \geq 0}$, with a constant arrival rate $\lambda_{j} \geq 0$ and random jump sizes $V^{j}$ having double exponential distribution $\nu_{j}(\mathrm{~d} y)$, i.e.

$$
\nu_{j}(\mathrm{~d} y)=\left(p_{j} \eta_{j 1} \mathrm{e}^{-\eta_{j 1} y} 1_{\{y \geq 0\}}+\left(1-p_{j}\right) \eta_{j 2} \mathrm{e}^{\eta_{j 2} y} 1_{\{y<0\}}\right) \mathrm{d} y,
$$

where $p_{j} \in[0,1], \eta_{j 1}>1$ and $\eta_{j 2}>0$. In addition, $\left(W_{t}^{j}\right)_{t \geq 0}$ and $\left(N_{t}^{j}\right)_{t \geq 0}$ are independent of the Markov chain $\left(J_{t}\right)_{t \geq 0}$.

From the standard theory of Lévy processes (see, for example, Cont and Tankov (2004)), the distributional properties of each Lévy process can be recovered from its own moment generating function. In fact, as shown in Kou (2002), the Lévy-Khintchine formula on $\left(X_{t}^{j}\right)_{t>0}$, for $j=1, \ldots, d$, admits an explicit form, i.e.

$$
\mathbb{E}\left[\exp \left(u X_{t}^{j}\right)\right]=\exp \left(\kappa_{j}(u) t\right), \quad \text { for } \quad t \geq 0, \quad u \in \mathbb{R} \backslash\left\{\eta_{j 1},-\eta_{j 2}\right\},
$$

where

$$
\kappa_{j}(u)=b_{j} u+\frac{\sigma_{j}^{2}}{2} u^{2}+\lambda_{j}\left(\frac{p_{j} \eta_{j 1}}{\eta_{j 1}-u}+\frac{\left(1-p_{j}\right) \eta_{j 2}}{\eta_{j 2}+u}-1\right) .
$$

We are now ready to give a precise definition of the regime-switching, double-exponential jump-diffusion process considered in this paper.

Definition 2.1. Define an asset price $S_{t}=S_{0} \exp \left(X_{t}\right)$, where the regime-switching, doubleexponential jump-diffusion process, denoted by $\left(X_{t}, J_{t}\right)_{t \geq 0}$, is defined pathwisely via

$$
\mathrm{d} X_{t} \triangleq \mathrm{d} X_{t}^{J_{t}}, X_{0} \triangleq X_{0}^{J_{0}}=0
$$

That is, conditional on $\left\{J_{t}=j\right\}, X_{t}^{j}$ is a double-exponential jump-diffusion process of the form in (2). 
By Definition 2.1, the regime-switching, double-exponential jump process $\left(X_{t}, J_{t}\right)_{t \geq 0}$ has the following canonical representation:

$$
X_{t}=X_{0}^{J_{0}}+\int_{0}^{t} b_{J_{s}} \mathrm{~d} s+\int_{0}^{t} \sigma_{J_{s}} \mathrm{~d} W_{s}^{J_{s}}+\sum_{j \in E} \int_{0}^{t} 1_{\left\{J_{s}=j\right\}} \mathrm{d} N_{s}^{j} .
$$

Direct application of Itô's Lemma gives the corresponding infinitesimal generator for $\left(X_{t}, J_{t}\right)_{t \geq 0}$, denoted by $\mathcal{A}$.

Lemma 2.2. Let $f: \mathbb{R} \times E \rightarrow \mathbb{R}$ be a function that is twice continuously differentiable in $\mathbb{R}$. Then, the infinitesimal generator for $\left(X_{t}, J_{t}\right)_{t \geq 0}$, denoted by $\mathcal{A}^{L}$, takes the form

$$
\begin{aligned}
\mathcal{A} f(x, i) \triangleq & \lim _{t \rightarrow 0} \frac{\mathbb{E}\left[f\left(X_{t}, J_{t}\right) \mid X_{0}=x, J_{0}=i\right]-f(x, i)}{t} \\
= & b_{i} \partial_{x} f(x, i)+\frac{1}{2} \sigma_{i}^{2} \partial_{x x} f(x, i) \\
& +\int_{\mathbb{R} \backslash\{0\}}(f(x+y, i)-f(x, i)) \lambda_{i} \nu_{i}(\mathrm{~d} y)+\sum_{j \in E} q_{i j} f(x, j) .
\end{aligned}
$$

Denote $\mathbb{Q}_{i}[.] \triangleq \mathbb{Q}\left[. \mid J_{0}=i\right]$ and $\mathbb{E}_{i}[.] \triangleq \mathbb{E}\left[. \mid J_{0}=i\right]$. Parallel to the case of jump-diffusion processes, the moment generating function describes the distributional properties of regimeswitching jump-diffusion process $\left(X_{t}, J_{t}\right)_{t \geq 0}$.

Proposition 2.3. Denote by $\mathbf{F}_{t}[u]$ the $d \times d$ matrix with $(i, j)^{\text {th }}$ element $\mathbb{E}_{i}\left[\mathrm{e}^{u X_{t}} ; J_{t}=j\right]$ and $u \in \mathbb{R} \backslash\left\{\eta_{j 1},-\eta_{j 2}, j=1, \ldots, d\right\}$.

Then, the moment generating function $F_{t}[u]$ for $\left(X_{t}, J_{t}\right)_{t \geq 0}$ in Definition 2.1 takes the form as follows:

$$
\mathbf{F}_{t}[u]=\mathrm{e}^{t \mathbf{K}[u]}
$$

where

$$
\mathbf{K}[u]=\mathbf{Q}+\mathbf{A}
$$

Here, $\mathbf{A} \triangleq\left\{\kappa_{j}(u)\right\}_{\text {diag }}$ denotes the $d \times d$ matrix in which $\mathbf{A}_{j j}=\kappa_{j}(u)$ and 0 otherwise. The Laplace exponent $\kappa_{j}(u)$ is given by (4).

Proof. Direct application of Proposition 2.1 in Asmussen (2003) to our model yields the desired result.

Since $\mathbb{Q}$ denotes the risk-neutral measure, every discounted price process is a martingale under $\mathbb{Q}$. To this end, we have the following martingale condition.

Denote $\mathbf{e}_{i}=(0, \cdots, 0,1,0, \cdots, 0)^{\top} \in \mathbb{R}^{d}$ the unit vector with 1 in the $i$-th component and $\mathbf{1}=(1, \cdots, 1)^{\top} \in \mathbb{R}^{d}$. Here, $\mathbf{b}^{\top}$ denotes the transpose of $\mathbf{b}$. Finally, let $\mathbf{I}$ be the $d \times d$ identity matrix. 
Lemma 2.4. Assume that $J_{t}=j$. The discounted process $\left(\bar{S}_{t}\right)_{t \geq 0} \triangleq\left(\mathrm{e}^{-r t} S_{t}\right)_{t \geq 0}$ is a $\mathbb{Q}$ martingale with respect to $\mathcal{F}_{t}$ if and only if

$$
b_{j}=r-\frac{1}{2} \sigma_{j}^{2}-\lambda_{j}\left(\zeta_{j}-1\right),
$$

where $r>0$ is the risk-free interest rate and

$$
\zeta_{j}=\frac{p_{j} \eta_{j 1}}{\eta_{j 1}-1}+\frac{\left(1-p_{j}\right) \eta_{j 2}}{\eta_{j 2}+1}
$$

Proof. Let $\left(\bar{X}_{t}^{j}\right)_{t \geq 0}$ be a jump-diffusion process with the triplet $\left(-r+b_{j}, \sigma_{j}^{2}, \nu_{j}\right)$ and the Lévy exponent $\bar{\kappa}_{j}(u)$, where

$$
\bar{\kappa}_{j}(u) \triangleq-u r+\kappa_{j}(u), u \in \mathbb{R} \backslash\left\{\eta_{j 1},-\eta_{j 2}\right\} .
$$

Following Definition 2.1, define the process $\mathrm{d} \bar{X}_{t} \triangleq \mathrm{d} \bar{X}_{t}^{J_{t}}$. Proposition 2.3 gives us that

$$
\begin{aligned}
\mathbb{E}\left[\exp \left(\bar{X}_{t}\right) \mid \mathcal{F}_{u}\right] & =\mathbb{E}\left[\exp \left(\bar{X}_{u}\right) \mathbf{e}_{J_{u}}^{\top} \exp \left(\bar{X}_{t}-\bar{X}_{u}\right) \mathbf{1} \mid \mathcal{F}_{u}\right] \\
& =\exp \left(\bar{X}_{u}\right) \mathbf{e}_{J_{u}}^{\top} \exp \left(\left(\mathbf{Q}+\left\{\bar{\kappa}_{k}(1)\right\}_{\text {diag }}\right)(t-u)\right) \mathbf{1}
\end{aligned}
$$

From (8), it becomes clear that $\bar{S}_{t}=\exp \left(\bar{X}_{t}\right)$ is an $\mathcal{F}_{t}$-martingale if and only if

$$
\mathbf{e}_{J_{u}}^{\top} \exp \left(\left(\mathbf{Q}+\left\{\bar{\kappa}_{k}(1)\right\}_{\text {diag }}\right)(t-u)\right) \mathbf{1}=1, \quad \text { for all } \quad u \leq t .
$$

We claim that $\bar{\kappa}_{j}(1)=0$ is a necessary and sufficient condition for (9) to hold for all $t \geq 0$ and $j \in E$. Suppose first that for all $j \in E$, we have

$$
\bar{\kappa}_{j}(1)=0, \quad t \geq 0 .
$$

By the definition of $\mathbf{Q}, \mathbf{Q}^{n} \mathbf{1}=\mathbf{0}$, where $n \in \mathbb{N}$. Hence $\mathbf{Q}$ has an eigenvalue of 0 with the eigenvector 1 . This implies that

$$
\exp \left(\left(\mathbf{Q}+\left\{\bar{\kappa}_{k}(1)\right\}_{\text {diag }}\right)(t-u)\right) \mathbf{1}=\exp (\mathbf{Q}(t-u)) \mathbf{1}=\mathbf{1} .
$$

Therefore, (9) holds. On the other hand, suppose that (9) holds. By, L'Hôpital's Rule, we have

$$
0=\lim _{u \downarrow t} \mathbf{e}_{J_{u}}^{\top} \frac{1}{t-u}\left[\exp \left(\left(\mathbf{Q}+\left\{\bar{\kappa}_{k}(1)\right\}_{\text {diag }}\right)(t-u)\right)-\mathbf{I}\right] \mathbf{1}=\mathbf{e}_{J_{t}}^{\top}\left(\mathbf{Q}+\left\{\bar{\kappa}_{k}(1)\right\}_{\text {diag }}\right) \mathbf{1}=\bar{\kappa}_{J_{t}}(1) .
$$

Since it must hold for any $J_{t}$, we have $\bar{\kappa}_{j}(1)=0$ for all $j \in E$ and $t \geq 0$. Therefore, the martingale property of $\left(\bar{S}_{t}\right)_{t \geq 0}=\left(\exp \left(\bar{X}_{t}\right)\right)_{t \geq 0}$ is equivalent to $\bar{\kappa}_{j}(1)=0$ for all $j \in E$ and $t \geq 0$.

When valuing the options, we encounter the issue of invertibility of the matrix $(a \mathbf{I}-\mathbf{K}[u])$, for $a \in \mathbb{R}$. To this end, Mijatović and Pistorius (2011) provided a sufficient condition for the inverse of $(a \mathbf{I}-\mathbf{K}[u])$ to exist, where $a \in \mathbb{C}$. It is reproduced in the following lemma, with a few modifications to fit into our framework. Throughout the rest of this paper, we denote $\Re(x)$ the real part of $x \in \mathbb{C}$. 
Lemma 2.5. (Mijatović and Pistorius, 2011)

Let $a \in \mathbb{C}$ and assume that $\Re(a)>\max \left\{\Re\left(\kappa_{i}(u)\right): i=1, \ldots, d\right\}$, then

$$
\mathbf{K}[u]-a \mathbf{I}=\mathbf{Q}-\left(a \mathbf{I}-\left\{\kappa_{i}(u)\right\}_{\text {diag }}\right)
$$

is invertible and

$$
\lim _{T \rightarrow \infty} \int_{0}^{T} \exp ((\mathbf{K}[u]-a \mathbf{I}) t) \mathrm{d} t=(a \mathbf{I}-\boldsymbol{K}[u])^{-1}
$$

As we shall see in Section 3, the valuations of the GMDBs can be greatly simplified in some cases when the underlying asset price process is the numéraire. In this respect, we conclude this section with the following proposition.

Proposition 2.6. Define $\tilde{\mathbb{Q}}$ to be an equivalent martingale measure of $\mathbb{Q}$ via the RadonNikodym derivative

$$
\left.\frac{\mathrm{d} \tilde{\mathbb{Q}}}{\mathrm{d} \mathbb{Q}}\right|_{\mathcal{F}_{t}}=\mathrm{e}^{-r t} \frac{S_{t}}{S_{0}}
$$

Let $\left(X_{t}, J_{t}\right)$ be the regime-switching, double-exponential jump-diffusion process in Definition 2.1 under measure $\mathbb{Q}$. Then, the process $\left(X_{t}, J_{t}\right)_{t \geq 0}$ remains a regime-switching, doubleexponential jump-diffusion process under $\tilde{\mathbb{Q}}$ with the moment generating function denoted by $\widehat{\mathbf{F}}_{t}[u]$ as follows.

For $u \in \mathbb{R} \backslash\left\{\tilde{\eta}_{j 1},-\tilde{\eta}_{j 2}, j=1, \ldots, d\right\}$,

$$
\widehat{\mathbf{F}}_{t}[u]=\mathrm{e}^{t \widehat{\mathbf{K}}[u]}
$$

where

$$
\widehat{\mathbf{K}}[u]=\mathbf{Q}+\left\{\tilde{\kappa}_{j}(u)\right\}_{\text {diag }},
$$

and the corresponding Lévy exponent $\tilde{\kappa}_{j}(u)$ is

$$
\tilde{\kappa}_{j}(u)=\tilde{b}_{j} u+\frac{\sigma_{j}^{2}}{2} u^{2}+\tilde{\lambda}_{j}\left(\frac{\tilde{p}_{j} \tilde{\eta}_{j 1}}{\tilde{\eta}_{j 1}-u}+\frac{\left(1-\tilde{p}_{j}\right) \tilde{\eta}_{j 2}}{\tilde{\eta}_{j 2}+u}-1\right)
$$

with $\tilde{b}_{j}=b_{j}+\sigma_{j}, \quad \tilde{\lambda}_{j}=\lambda_{j}\left(\zeta_{j}+1\right), \quad \tilde{p}_{j}=\frac{p_{j} \eta_{j 1}}{\left(\zeta_{j}+1\right)\left(\eta_{j 1}-1\right)}, \quad \tilde{\eta}_{j 1}=\eta_{j 1}-1, \quad \tilde{\eta}_{j 2}=\eta_{j 2}+1$, and $\zeta_{j}$ takes the form in (7).

Proof. One can either follow the proof in Proposition 2.1 of Asmussen (2003), together with Proposition 9.8 of Cont and Tankov (2004), or directly apply Theorem 4.3.1 of Siu (2012) to our model to obtain the desired result.

\section{$2.2 \quad$ First passage time distribution of $\left(X_{t}, J_{t}\right)_{t>0}$}

As it will become clear in the following sections, valuing certain life-contingent contracts hinges on the knowledge of the distribution of the maximum or the minimum of the asset 
price over the life of the contract. This implies that we need to know the distribution of the first passage time of the asset price. More specifically, we are interested in finding the distribution of the first time when $X_{t}$ exits a pre-specified interval. Denote $\tau_{B}$ the first exit time of $X_{t}$ from an interval $[L, U]$, i.e.

$$
\tau_{B} \triangleq \inf \left\{t \geq 0: X_{t} \notin[L, U]\right\}
$$

where $L<X_{0}=x<U$ and $J_{0}=i$.

It is easy to see that finding the first passage time distribution is equivalent to solving $\mathbb{E}_{i}\left[\mathrm{e}^{-a \tau_{B}+b X_{\tau_{B}}}\right]$ by the optional stopping theorem, which admits an explicit structure under our model. See also Asmussen et al. (2004) for the two-regime case, i.e. $E=\{1,2\}$.

To compute $\mathbb{E}_{i}\left[\mathrm{e}^{-a \tau_{B}+b X_{\tau_{B}}}\right]$, we first transform the original process $\left(X_{t}, J_{t}\right)_{t>0}$ into a fluid process $\left(\tilde{X}_{t}, \tilde{J}_{t}\right)_{t \geq 0}$ by removing the jumps in the sample path of $\left(X_{t}, J_{t}\right)_{t \geq 0}$. For the ease of exposition, we shall only state the representation of the first passage time distribution of $\left(X_{t}, J_{t}\right)_{t \geq 0}$ (Theorem 2.7 and Corollary 2.8) in the remainder of the section. See Appendix A for the construction of the fluid process $\left(\tilde{X}_{t}, \tilde{J}_{t}\right)_{t \geq 0}$ and the corresponding proofs.

Define

$$
\tilde{\mathbf{K}}_{a}[u]=\left(\begin{array}{cccc}
\tilde{\mathbf{K}}_{a}^{1}[u] & \mathbf{O} & \cdots & \mathbf{O} \\
\mathbf{O} & \tilde{\mathbf{K}}_{a}^{2}[u] & \cdots & \mathbf{O} \\
\vdots & & \ddots & \vdots \\
\mathbf{O} & \cdots & \mathbf{O} & \tilde{\mathbf{K}}_{a}^{d}[u]
\end{array}\right)+\left(\begin{array}{cccc}
\tilde{\mathbf{Q}}_{11} & & \cdots & \tilde{\mathbf{Q}}_{1 d} \\
\vdots & & \ddots & \vdots \\
\tilde{\mathbf{Q}}_{d 1} & \cdots & & \tilde{\mathbf{Q}}_{d d}
\end{array}\right)
$$

where

$$
\tilde{\mathbf{K}}_{a}^{j}[u] \triangleq\left(\begin{array}{ccc}
b_{j} u+\frac{\sigma_{j}^{2}}{2} u^{2}-\lambda_{j}-a & \lambda_{j}\left(1-p_{j}\right) & \lambda_{j} p_{j} \\
\eta_{j 2} & \eta_{j 2}+u & 0 \\
\eta_{j 1} & 0 & \eta_{j 1}-u
\end{array}\right), \quad j=1, \ldots, d
$$

and

$$
\tilde{\mathbf{Q}}_{i i}=\left(\begin{array}{ccc}
q_{i i} & 0 & 0 \\
0 & 0 & 0 \\
0 & 0 & 0
\end{array}\right), \quad \tilde{\mathbf{Q}}_{i j}=\left(\begin{array}{ccc}
q_{i j} & 0 & 0 \\
0 & 0 & 0 \\
0 & 0 & 0
\end{array}\right), \quad i, j=1, \ldots, d
$$

By Lemma A.5, we can denote $\varrho_{l, a}$, for $l=1, \ldots, 4 d$, to be the distinct real roots of the equation

$$
\operatorname{det}\left(\tilde{\mathbf{K}}_{a}[u]\right)=0
$$

for each $\varrho_{l, a}$, let

$$
\mathbf{h}^{l}[a]=\left(\begin{array}{c}
\mathbf{h}_{1}^{l}[a] \\
\vdots \\
\mathbf{h}_{d}^{l}[a]
\end{array}\right), \quad \mathbf{h}_{j}^{l}[a]=\left(\begin{array}{c}
h_{(j, 0)}^{l}[a] \\
h_{(j,-)}^{l}[a] \\
h_{(j,+)}^{l}[a]
\end{array}\right), \quad j=1, \ldots, d
$$


be the basis of the kernel of $\tilde{\mathbf{K}}_{a}^{X}\left[\varrho_{l, a}\right]$, for $l=1, \ldots, 4 d$. With (13), define a $4 d \times 4 d$ matrix $\mathbf{H}[a]$ as follows:

$$
\mathbf{H}[a] \triangleq\left(\begin{array}{ccc}
\tilde{\mathbf{h}}_{1}^{1}[a] & \ldots & \tilde{\mathbf{h}}_{d}^{1}[a] \\
\vdots & \ddots & \vdots \\
\tilde{\mathbf{h}}_{1}^{4 d}[a] & \ldots & \tilde{\mathbf{h}}_{d}^{4 d}[a]
\end{array}\right)
$$

and $\tilde{\mathbf{h}}_{j}^{l}[a]$ is a $4 \times 1$ vector of the form,

$$
\tilde{\mathbf{h}}_{j}^{l}[a] \triangleq\left(\mathrm{e}^{\varrho_{l, a} U} h_{(j, 0)}^{l}[a], \quad \mathrm{e}^{\varrho_{l, a} U} h_{(j,+)}^{l}[a], \mathrm{e}^{\varrho_{l, a} L} h_{(j, 0)}^{l}[a], \mathrm{e}^{\varrho_{l, a} L} h_{(j,-)}^{l}[a]\right),
$$

where $l=1, \ldots, 4 d$, and $j=1, \ldots, d$.

Let

$$
\mathbf{x}[a] \triangleq\left(\mathrm{e}^{\varrho_{1, a} x} h_{(i, 0)}^{1}[a], \ldots, \mathrm{e}^{\varrho_{4 d, a} x} h_{(i, 0)}^{4 d}[a]\right)^{\top} .
$$

The following theorem provides the representation of $\mathbb{E}_{i}\left[\mathrm{e}^{-a \tau_{B}}\right]$.

Theorem 2.7. Assume that $J_{0}=i$, and

$$
-\infty<-\eta_{d 2}<\ldots<-\eta_{12}<0<\eta_{11}<\ldots<\eta_{d 1}<\infty .
$$

Then, for any $a>0$,

$$
\mathbb{E}_{i}\left[\mathrm{e}^{-a \tau_{B}}\right]=\boldsymbol{\pi}[a]^{\top} \mathbf{1},
$$

where the $4 d \times 1$ vector $\boldsymbol{\pi}[a]$ is the solution of the following system of linear equations:

$$
\mathbf{x}[a]=\mathbf{H}[a] \boldsymbol{\pi}[a],
$$

where $\mathbf{H}[a]$ and $\mathbf{x}[a]$ are defined in (14) and (16), respectively.

Proof. See Appendix A.3 for a proof.

To compute $\mathbb{E}_{i}\left[\mathrm{e}^{-a \tau_{B}+b X_{\tau_{B}}} 1_{\left\{J_{\tau_{B}}=j\right\}}\right]$, we can invoke the properties of conditional independence and memoryless in Corollary A.2 and obtain the following corollary.

Corollary 2.8. Let $\widehat{\mathbf{f}}$ be a $4 d \times 1$ vector of the following form,

$$
\widehat{\mathbf{f}}[b] \triangleq\left(\widehat{f}_{(1,0)}^{U}[b], \widehat{f}_{(1,+)}^{U}[b], \ldots, \widehat{f}_{(d, 0)}^{L}[b], \widehat{f}_{(d,-)}^{L}[b]\right)^{\top},
$$

where

$$
\begin{array}{ll}
\widehat{f}_{(j, 0)}^{U}[b] \triangleq \mathrm{e}^{b U}, & \widehat{f}_{(j,+)}^{U}[b] \triangleq \frac{\eta_{j 1}}{\eta_{j 1}-b} \mathrm{e}^{b U}, \\
\widehat{f}_{(j, 0)}^{L}[b] \triangleq \mathrm{e}^{b L}, & \widehat{f}_{(j,-)}^{L}[b] \triangleq \frac{\eta_{j 2}}{\eta_{j 2}+b} \mathrm{e}^{b L} .
\end{array}
$$

Then,

$$
\mathbb{E}_{i}\left[\mathrm{e}^{-a \tau_{B}+b X_{\tau_{B}}}\right]=\boldsymbol{\pi}[a]^{\top} \widehat{\mathbf{f}}[b],
$$

where $J_{0}=i, X_{0}=x$, and $\boldsymbol{\pi}[a]$ is the solution in Theorem 2.7. 
Proof. See Appendix A.3 for a proof.

Example 2.1. Consider the case of two regimes, i.e. $E=\{1,2\}$. Let $\gamma_{l}=\frac{\kappa_{2}\left(\varrho_{l, a}\right)-a-q_{22}}{q_{22}}, l=$ $1, \ldots, 8$, where the $\varrho_{l, a}$ 's are obtained solving the following equation

$$
0=\operatorname{det}\left(\tilde{\mathbf{K}}_{a}[u]\right)=\left(\kappa_{1}(u)-a-q_{11}\right)\left(\kappa_{2}(u)-a-q_{22}\right)-q_{11} q_{22} .
$$

In addition, in this case, the basis of the kernel of $\left\{\boldsymbol{h}^{l}[a]\right\}_{l=1, \ldots, 8}$, admits an explicit form as follows. For each $l=1, \ldots, 8$, it follows that

$$
\boldsymbol{h}^{l}[a]=\left(\begin{array}{c}
\boldsymbol{h}_{1}^{l}[a] \\
\boldsymbol{h}_{2}^{l}[a]
\end{array}\right), \boldsymbol{h}_{1}^{l}[a]=\left(\begin{array}{c}
\gamma_{l} \\
\frac{\gamma_{r} \eta_{12}}{\eta_{12}+\varrho_{l, a}} \\
\frac{\gamma_{r} \eta_{11}}{\eta_{11}-\varrho_{l, a}}
\end{array}\right), \boldsymbol{h}_{2}^{l}[a]=\left(\begin{array}{c}
-1 \\
\frac{-\eta_{22}}{\eta_{22}+\varrho_{l, a}} \\
\frac{-\eta_{21}}{\eta_{21}-\varrho_{l, a}}
\end{array}\right)
$$

Applying Corollary 2.8 to (20), we can then obtain the explicit representations of $\mathbb{E}_{i}\left[\mathrm{e}^{-a \tau_{B}+b X_{\tau_{B}}}\right]$.

\subsection{Time-until-death $T_{\mathrm{x}}$}

As discussed in the introduction, Botta and Harris (1986) showed that the family of all combinations of exponential distributions approximates any probability distribution on the positive axis with arbitrary accuracy. The combination of exponential distributions has also found its place in mortality modeling. In particular, Dufresne (2007) used combinations of exponentials to construct the Jacobi polynomial expansion and logbeta distribution schemes to approximate many well-known distributions in actuarial literature. In light of these results, it suffices for us to consider the problems of valuing equity-death benefits under the assumption that the time-until-death random variable is exponentially distributed, i.e. with density function given in (21) below. In Section 5.1, we revisit the valuations of the equity-death benefits using combinations of the exponential functions calibrated to a life table.

Denote by $T_{\mathrm{x}}$ the time-until-death random variable for a person of age $\mathrm{x}$, independent of the price process $\left(S_{t}\right)_{t \geq 0}$. Based on the above discussion, we assume that $T_{\mathrm{x}}$ is exponentiallydistributed with probability density function

$$
f_{T_{\mathrm{x}}}(t)=\alpha \mathrm{e}^{-\alpha t}, \quad \alpha>0, t>0
$$

where $\alpha$ denotes the force of mortality.

\section{Application to valuations of different options}

As in Gerber et al. (2012, 2013), we are interested in valuing embedded options of the form

$$
\mathbb{E}\left[\mathrm{e}^{-r T_{\mathrm{x}}} b\left(T_{\mathrm{x}}, S_{T_{\mathrm{x}}}\right)\right]
$$


where $b$ is called an equity-indexed benefit function. Since the time-until-death random variable of an individual at age $\mathrm{x}, T_{\mathrm{x}}$, is independent of the stock price process $S_{t},(22)$ becomes

$$
\mathbb{E}\left[\mathrm{e}^{-r T_{\mathrm{x}}} b\left(T_{\mathrm{x}}, S_{T_{\mathrm{x}}}\right)\right]=\int_{0}^{\infty} \mathbb{E}\left[\mathrm{e}^{-r T} b\left(T, S_{T}\right)\right] f_{T_{\mathrm{x}}}(T) \mathrm{d} T=\alpha \int_{0}^{\infty} \mathbb{E}\left[\mathrm{e}^{-r T} b\left(T, S_{T}\right)\right] \mathrm{e}^{-\alpha T} \mathrm{~d} T .
$$

The above representation is indeed the Laplace (Carson) transform of $\mathbb{E}\left[\mathrm{e}^{-r T} b\left(T, S_{T}\right)\right]$ with respect to the maturity $T$, evaluated at the force of mortality $\alpha$.

We denote by $\mathcal{L}_{\varphi}(\cdot)$ and $\mathcal{L}_{k}^{-1}(\cdot)$ the Laplace transform and inverse Laplace transform operators with respect to $\varphi$ and $k$, respectively. To recover the value of each option, we need to perform a numerical Laplace inversion. We shall adopt the Abate-Whitt algorithm, first proposed by Abate and Whitt (1992) on $\mathbb{R}_{+}$and later extended by Petrella (2004) to $\mathbb{R}$. The basic ideas behind the Abate-Whitt algorithm are delegated in Appendix C.

\subsection{Basic options}

As discussed in the introduction, finding the fair value of the basic option with the payment of $\max \left(S_{T_{\mathrm{x}}}, K\right)$ to the beneficiary of the policy rider upon the policyholder's death $T_{\mathrm{x}}$ amounts to computing the fair (discounted) value of the option with the equity-indexed benefit function given as follows:

$$
b\left(T_{\mathrm{x}}, S_{T_{\mathrm{x}}}\right)=\left(K-S_{T_{\mathrm{x}}}\right)^{+} .
$$

To value this put option, the equity-indexed benefit function $b\left(T_{\mathrm{x}}, S_{T_{\mathrm{x}}}\right)$ indicates that only the knowledge of the distribution of the asset price at the time $T_{\mathrm{x}}$ is required. In the Laplace transform framework, we have the following result.

Theorem 3.1. Let $T_{\mathrm{x}}$ be the time-until-death random variable with the density function (21).

For all $\varphi$ satisfying $0<\varphi<\min _{j \in E}\left\{\eta_{j 1}-1\right\}$ and $\alpha+r>\max _{j \in E}\left\{\kappa_{j}(\varphi+1)\right\}$, where $\kappa_{j}(u)$ is given in (4), the value of the basic call option, denoted by $C\left(K, T_{\mathrm{x}}, i\right)$, takes the form

$$
\begin{aligned}
C\left(K, T_{\mathrm{x}}, i\right) & =\mathbb{E}_{i}\left[\mathrm{e}^{-r T_{\mathrm{x}}}\left(S_{T_{\mathrm{x}}}-K\right)^{+}\right] \\
& =\mathcal{L}_{k}^{-1}\left(\frac{\alpha \chi^{-\varphi} S_{0}^{\varphi+1}}{\varphi(\varphi+1)} \mathbf{e}_{i}^{\top}((\alpha+r) \mathbf{I}-\mathbf{K}[\varphi+1])^{-1} \mathbf{1}\right),
\end{aligned}
$$

where $k=-\log (K / \chi)$ and $\chi>K$ is the scaling parameter. ${ }^{3}$ The value of the basic put option, denoted by $P\left(K, T_{\mathrm{x}}, i\right)$, takes the form

$$
\begin{aligned}
P\left(K, T_{\mathrm{x}}, i\right) & =\mathbb{E}_{i}\left[\mathrm{e}^{-r T_{\mathrm{x}}}\left(K-S_{T_{\mathrm{x}}}\right)^{+}\right] \\
& =\mathcal{L}_{k}^{-1}\left(\frac{\alpha \chi^{-\varphi} S_{0}^{\varphi+1}}{\varphi(\varphi+1)} \mathbf{e}_{i}^{\top}((\alpha+r) \mathbf{I}-\mathbf{K}[\varphi+1])^{-1} \mathbf{1}\right)-S_{0}+\frac{\alpha}{\alpha+r} K .
\end{aligned}
$$

\footnotetext{
${ }^{3}$ As discussed in Petrella (2004) and Cai and Kou (2011), the presence of the scaling parameter $\chi$ makes the bilateral Abate-Whitt algorithm on $\mathbb{R}$ faster and more stable than the original Abate-Whitt algorithm. We make use of the scaling parameter $\chi$ when we perform bilateral Laplace transforms, i.e. Laplace transforms on the entire real line $\mathbb{R}$. See Appendix $\mathrm{C}$ for the discussion on the role of $\chi$ in the numerical implementations.
} 
Proof. We first prove (24).

$$
\begin{aligned}
& \int_{-\infty}^{\infty} \mathrm{e}^{-\varphi k} \chi \mathbb{E}_{i}\left[\mathrm{e}^{-r T_{\mathrm{x}}}\left(\frac{S_{0}}{\chi} \mathrm{e}^{X_{T_{\mathrm{x}}}}-\mathrm{e}^{-k}\right)^{+}\right] \mathrm{d} k \\
= & \int_{0}^{\infty} \alpha \mathrm{e}^{-(\alpha+r) T} \chi \mathbb{E}_{i}\left[\int_{-\log \left(\frac{S_{0}}{\chi} \mathrm{e}^{X_{T}}\right)}^{\infty} \mathrm{e}^{-\varphi k}\left(\frac{S_{0}}{\chi} \mathrm{e}^{X_{T}}-\mathrm{e}^{-k}\right) \mathrm{d} k\right] \mathrm{d} T \\
= & \chi\left(\frac{S_{0}}{\chi}\right)^{\varphi+1} \int_{0}^{\infty} \alpha \mathrm{e}^{-(\alpha+r) T} \sum_{j \in E} \mathbb{E}_{i}\left[\frac{\mathrm{e}^{(\varphi+1) X_{T}}}{\varphi(\varphi+1)} 1_{\left\{J_{T_{\mathrm{x}}}=j\right\}}\right] \mathrm{d} T \\
= & \chi\left(\frac{S_{0}}{\chi}\right)^{\varphi+1} \frac{\alpha}{\varphi(\varphi+1)} \mathbf{e}_{i}^{\top}\left(\int_{0}^{\infty} \mathrm{e}^{-((\alpha+r) \mathbf{I}-\mathbf{K}[\varphi+1]) T} \mathrm{~d} T\right) \mathbf{1} \\
= & \chi\left(\frac{S_{0}}{\chi}\right)^{\varphi+1} \frac{\alpha}{\varphi(\varphi+1)} \mathbf{e}_{i}^{\top}((\alpha+r) \mathbf{I}-\mathbf{K}[\varphi+1])^{-1} \mathbf{1},
\end{aligned}
$$

where we makes use of the Fubini Theorem twice in the first equality, Proposition 2.3 and the independence assumption between $S_{t}$ and $T_{\mathrm{x}}$, for all $t \geq 0$, in the fourth equality, and Lemma 2.5 in the last equality. The price of the call option $C\left(K, T_{\mathrm{x}}, i\right)$ then follows by taking the inverse Laplace transform of (26) with respect to $k$. Finally, the price of the put option $P\left(K, T_{\mathrm{x}}, i\right)$ can be obtained by combining $(24)$ with the put-call parity:

$$
C\left(K, T_{\mathrm{x}}, i\right)-P\left(K, T_{\mathrm{x}}, i\right)=S_{0}-K \mathbb{E}\left[\mathrm{e}^{-r T_{\mathrm{x}}}\right]=S_{0}-\frac{\alpha}{\alpha+r} K
$$

Observe that Theorem 3.1 covers out-of-money, at-the-money, and in-the-money call options. Moreover, the proof of Theorem 3.1 also includes the pricing of all-or-nothing options. As discussed Gerber et al. (2012, 2013), the valuation of the basic options in Theorem 3.1 can be extended to accommodate additional contractual features. For example, consider the basic option with roll-up or rising-floor feature, discussed in Milesvsky and Posner (2001) and Ulm (2006, 2008). Following the notations in $\operatorname{Ulm}(2006,2008)$, we let $p$ to be the "roll-up" rate such that $0 \leq p \leq r$ to preclude any arbitrage opportunities. Then, the roll-up GMDB has the following equity-indexed benefit function

$$
b_{\text {roll-up }}\left(T_{\mathrm{x}}, S_{T_{\mathrm{x}}}\right) \triangleq\left(K \mathrm{e}^{p T_{\mathrm{x}}}-S_{T_{\mathrm{x}}}\right)^{+} .
$$

Since (26) can be rewritten as

$$
\left(K \mathrm{e}^{p T_{\mathrm{x}}}-S_{T_{\mathrm{x}}}\right)^{+}=\mathrm{e}^{p T_{\mathrm{x}}}\left(K-\mathrm{e}^{-p T_{\mathrm{x}}} S_{T_{\mathrm{x}}}\right)^{+},
$$

we can simply apply Theorem 3.1 to value the roll-up put option in (26) with $\widehat{S}_{T_{\mathrm{x}}}=\mathrm{e}^{-p T_{\mathrm{x}}} S_{T_{\mathrm{x}}}$ and discount factor equals $\mathrm{e}^{-(r-p) T_{\mathrm{x}}}$.

On the other hand, let us consider the basic option with the possibility of lapses or policy surrenders. We first assume that the decision of lapses or surrenders is independent of both the mortality random variable $T_{\mathrm{x}}$ and the asset price process $S_{t}$ (see $\left.\operatorname{Ulm}(2006,2008)\right) .{ }^{4}$

\footnotetext{
${ }^{4}$ We defer the discussion of the valuation of the options in which the decisions of lapses or surrenders depend on the path of the underlying asset to Section 3.3.
} 
Consider the case of a deterministic, non-increasing function $g(t)$ such that, given $T_{\mathrm{x}}=t$, $g(t)$ denotes the probability that the policy has not been lapsed by time $t$. Hence, the GMDB with deterministic lapse rate has the following equity-indexed benefit function

$$
b_{\text {lapse }}\left(T_{\mathrm{x}}, S_{T_{\mathrm{x}}}\right) \triangleq g\left(T_{\mathrm{x}}\right)\left(K-S_{T_{\mathrm{x}}}\right)^{+} .
$$

If $g(t)=\mathrm{e}^{-\nu t}$, with some constant $\nu>0$ denoting the force of surrender, the fair value of the GMDB with equity-indexed benefit function in (27) becomes

$$
\mathbb{E}\left[\mathrm{e}^{-(r+\nu) T_{\mathrm{x}}} b_{\text {lapse }}\left(T_{\mathrm{x}}, S_{T_{\mathrm{x}}}\right)\right] .
$$

We can then apply Theorem 3.1 by replacing $r$ with $\widehat{r}=r+\nu$.

\subsection{Lookback options}

Instead of considering a constant minimum guaranteed amount $K$, as is the case in Section 3.1, let us analyze the GMDB with the guaranteed minimum amount being the running maximum of the underlying asset $S_{t}$ at any time $t$, i.e. $K=\max _{0 \leq s \leq t} S_{s}$. That is, the equity-indexed benefit function for this option at $T_{\mathrm{x}}$ becomes

$$
b_{\text {lookback }}\left(T_{\mathrm{x}}, S_{T_{\mathrm{x}}}\right) \triangleq \max _{0 \leq s \leq T_{\mathrm{x}}} S_{s}-S_{T_{\mathrm{x}}}
$$

This can be achieved by selling and then repurchasing the contract at the new level. The GMDB with the running maximum of the asset price as the guaranteed minimum amount is called lookback option and has been considered in Milevsky and Posner (2001) and Gerber et al. (2012) under the Brownian motion framework, and Gerber et al. (2013) under the jump-diffusion framework. In this section, we consider the fair value of the lookback option under the regime-switching jump-diffusion framework.

\subsubsection{Standard lookback options}

Since the lookback option with the equity-indexed benefit function in (28) constitutes a floating strike lookback put option, we begin our discussion with the valuation of a floatingstrike lookback put option. Other situations, such as fixed strike lookback call/put options, can be treated analogously. Let $M$ be the initial maximum of the asset price, then the floating-strike lookback put is given as

$$
\begin{aligned}
L P\left(S_{0}, M, T_{\mathrm{x}}, i\right) & \triangleq \mathbb{E}_{i}\left[\mathrm{e}^{-r T_{\mathrm{x}}}\left(\max \left(M, \max _{0 \leq t \leq T_{\mathrm{x}}} S_{t}\right)-S_{T_{\mathrm{x}}}\right)\right] \\
& =S_{0} \mathbb{E}_{i}\left[\mathrm{e}^{-r T_{\mathrm{x}}} \max \left(\mathrm{e}^{z}, \mathrm{e}^{M_{T_{\mathrm{x}}}^{X}}\right)\right]-S_{0} \\
& =S_{0} \mathbb{E}_{i}\left[\mathrm{e}^{-r T_{\mathrm{x}}}\left(\mathrm{e}^{M_{T_{\mathrm{x}}}^{X}}-\mathrm{e}^{z}\right) 1_{\left\{M_{T_{\mathrm{x}}}^{X} \geq z\right\}}\right]+S_{0} \mathrm{e}^{z} \mathbb{E}\left[\mathrm{e}^{-r T_{\mathrm{x}}}\right]-S_{0},
\end{aligned}
$$

where $z \triangleq \log \left(M / S_{0}\right), M_{T_{\mathrm{x}}}^{X} \triangleq \sup _{t \leq T_{\mathrm{x}}} X_{t}$, and $J_{0}=i$. Under the regime-switching jumpdiffusion framework, the value of the standard lookback put option in (29) is given as follows:

Theorem 3.2. Let $T_{\mathrm{x}}$ be the time-until-death random variable with the density function (21). 
The value of the standard lookback option is

$$
L P\left(S_{0}, M, T_{\mathrm{x}}, i\right)=\frac{\alpha S_{0}}{\alpha+r} \int_{z}^{\infty} \mathrm{e}^{m} \sum_{j} \mathbb{E}_{i}\left[\mathrm{e}^{-(\alpha+r) \tau_{m}} 1_{\left\{J_{\tau_{m}}=j\right\}}\right] \mathrm{d} m+\frac{M \alpha}{\alpha+r}-S_{0}
$$

where

$$
\tau_{m} \triangleq \inf \left\{t \geq 0: X_{t} \geq m\right\}
$$

To facilitate the proof of this theorem, we need the following two lemmas.

Lemma 3.3.

$$
\lim _{m \rightarrow \infty} \mathrm{e}^{m} \mathbb{Q}_{i}\left[M_{T}^{X} \geq m\right]=0, \text { for all } T \geq 0
$$

Proof. Let $\theta \in\left(\max _{j \in E}\left\{-\eta_{j 2}\right\}, \min _{j \in E}\left\{\eta_{j 1}\right\}\right)$. Observe that

$$
\left\{\exp \left(\theta X_{t}-\kappa_{J_{t}}(\theta) t\right)\right\}_{t \geq 0}
$$

is a martingale. Fix $\theta \in\left(1, \min _{j \in E}\left\{\eta_{j 1}\right\}\right)$ such that $\kappa_{J_{t}}(\theta)>0$. Such a $\theta$ always exists as $\kappa_{J_{t}}(\theta)$ is continuous on $\left(1, \min _{j \in E}\left\{\eta_{j 1}\right\}\right)$ and since $\kappa_{J_{t}}(1)=r_{J_{t}} \geq 0$ (see (4)). It follows that

$$
\begin{aligned}
\mathrm{e}^{m} \mathbb{Q}_{i}\left[M_{T}^{X} \geq m\right] & =\mathrm{e}^{(1-\theta) m} \mathrm{e}^{\theta m} \mathbb{Q}_{i}\left[M_{T}^{X} \geq m\right]=\mathrm{e}^{(1-\theta) m} \mathrm{e}^{\theta m} \mathbb{Q}_{i}\left[\tau_{m} \leq T\right] \\
& \leq \mathrm{e}^{(1-\theta) m} \mathbb{E}_{i}\left[\mathrm{e}^{\left.\theta X_{\tau_{m} \wedge T}\right]}\right. \\
& \leq \mathrm{e}^{(1-\theta) m} \mathrm{e}^{\kappa_{J_{T}}(\theta) T} \mathbb{E}_{i}\left[\exp \left(\theta X_{\tau_{m} \wedge T}-\kappa_{J_{T \wedge \tau_{m}}}(\theta)\left(T \wedge \tau_{m}\right)\right)\right] \\
& =\mathrm{e}^{(1-\theta) m} \mathrm{e}^{\kappa_{J_{T}}(\theta) T},
\end{aligned}
$$

where the last equality follows from the Doob's optional sampling theorem.

Note that we can replace the $T$ in Lemma 3.3 with $T_{\mathrm{x}}$ since $T_{\mathrm{x}}$ is independent of $S_{t}$, for all $t \geq 0$. To value the desired lookback option, closer observation reveals that one can decompose the lookback option into the sum of two digital options, which are detailed in following lemma:

\section{Lemma 3.4.}

$$
\mathrm{e}^{-r T} \mathbb{E}_{i}\left[\mathrm{e}^{M_{T}^{X}} 1_{\left\{M_{T}^{X} \geq z\right\}}\right]=\mathrm{e}^{-r T} \mathbb{E}_{i}\left[\mathrm{e}^{z} 1_{\left\{M_{T}^{X} \geq z\right\}}\right]+\mathrm{e}^{-r T} \int_{z}^{\infty} \mathrm{e}^{m} \mathbb{Q}_{i}\left[M_{T_{\mathrm{x}}}^{X} \geq m\right] \mathrm{d} m .
$$

Proof.

$$
\begin{aligned}
\mathrm{e}^{-r T} \mathbb{E}_{i}\left[\mathrm{e}^{M_{T}^{X}} 1_{\left\{M_{\tau}^{X} \geq z\right\}}\right] & =-\mathrm{e}^{-r T} \int_{z}^{\infty} \mathrm{e}^{m} d \mathbb{Q}_{i}\left[M_{T}^{X} \geq m\right] \\
& =-\mathrm{e}^{-r T}\left\{-\mathrm{e}^{z} \mathbb{Q}_{i}\left[M_{T}^{X} \geq z\right]-\int_{z}^{\infty} \mathrm{e}^{m} \mathbb{Q}_{i}\left[M_{T}^{X} \geq m\right] \mathrm{d} m\right\} \\
& =\mathrm{e}^{-r T} \mathbb{E}_{i}\left[\mathrm{e}^{z} 1_{\left\{M_{T}^{X} \geq z\right\}}\right]+\mathrm{e}^{-r T} \int_{z}^{\infty} \mathrm{e}^{m} \mathbb{Q}_{i}\left[M_{T}^{X} \geq m\right] \mathrm{d} m,
\end{aligned}
$$

where in the second equality we first perform integration by parts and then make use of the fact that $\lim _{m \rightarrow \infty} \mathrm{e}^{m} \mathbb{Q}_{i}\left[M_{T}^{X} \geq m \mid T_{\mathrm{x}}=T\right]=0$, proved in Lemma 3.3. 
With the necessary results in place, we are now in position to prove Theorem 3.2 .

Proof of Theorem 3.2. By the Fubini Theorem and Lemma 3.4, we see that

$$
L P\left(S_{0}, M, T_{\mathrm{x}}, i\right)=S_{0} \int_{z}^{\infty} \mathrm{e}^{m} \int_{0}^{\infty} \alpha \mathrm{e}^{-(\alpha+r) T} \mathbb{Q}_{i}\left[M_{T}^{X} \geq m\right] \mathrm{d} T \mathrm{~d} m+\frac{M \alpha}{\alpha+r}-S_{0} .
$$

Since $\mathbb{Q}_{i}\left[M_{T}^{X} \geq m\right]=\mathbb{Q}_{i}\left[\tau_{m}<T\right]$, we have

$$
\int_{0}^{\infty} \mathrm{e}^{-(\alpha+r) T} \mathbb{Q}_{i}\left[M_{T}^{X} \geq m\right] \mathrm{d} T=\int_{0}^{\infty} \mathrm{e}^{-(\alpha+r) T} \mathbb{Q}_{i}\left[\tau_{m}<T\right] \mathrm{d} T
$$

Invoking the Fubini Theorem once more, we have

$$
\int_{0}^{\infty} \mathrm{e}^{-(\alpha+r) T} \mathbb{Q}_{i}\left[\tau_{m}<T\right] \mathrm{d} T=\frac{1}{\alpha+r}\left[\sum_{j \in E} \mathbb{E}_{i}\left[\mathrm{e}^{-(\alpha+r) \tau_{m}} 1_{\left\{J_{\tau_{m}}=j\right\}}\right]\right]
$$

Since $T_{\mathrm{x}}$ and $S_{t}$ are mutually independent for all $t \geq 0$, it follows that

$$
L P\left(S_{0}, M, T_{\mathrm{x}}, i\right)=\frac{\alpha S_{0}}{\alpha+r} \int_{z}^{\infty} \mathrm{e}^{m} \sum_{j \in E} \mathbb{E}_{i}\left[\mathrm{e}^{-(\alpha+r) \tau_{m}} 1_{\left\{J_{\tau_{m}}=j\right\}}\right] \mathrm{d} m+\frac{M \alpha}{\alpha+r}-S_{0} .
$$

To complete the remaining computation, we need to know how to evaluate $\mathbb{E}_{i}\left[\mathrm{e}^{-(\alpha+r) \tau_{m}} 1_{\left\{J_{\tau_{m}}=j\right\}}\right]$, which is covered in Section A.3. Unlike the case of the basic options in Section 3.1, Theorem 3.2 involves no use of the inverse Laplace transform to recover the standard lookback options within our model.

Remark 3.5. In Gerber et al. (2012, 2013), the valuation of fractional lookback option follows naturally through the discounted densities, thanks to the property of stationary increments of Lévy processes. The presence of the regime-switching factor, i.e. the Markov chain $\left(J_{t}\right)_{t \geq 0}$, complicates the problem significantly as the property of stationary increments no longer holds. The usual strategy is to perform the change-of-numéraire technique in Proposition 2.6. Yet, the change-of-numéraire technique is applicable to the pricing of the fractional lookback options under the regime-switching framework only when the Markov chain $\left(J_{t}\right)_{t \geq 0}$ is reversible. However, a reversible Markov chain is a special form of Markov chain. For this reason, we choose not to discuss the valuation of fractional lookback options in this section, but rather defer it to Appendix B, where we shall solve the valuation problem under the additional assumption that the Markov chain $\left(J_{t}\right)_{t \geq 0}$ is reversible.

\subsection{Barrier options}

Let us now revisit the GMDB with the possibility of lapses or surrenders in Ulm $(2006,2008)$, i.e. the GMDB with the equity-indexed benefit function at the death of the investor, $T_{\mathrm{x}}$, as follows:

$$
b_{\text {lapse }}\left(T_{\mathrm{x}}, S_{T_{\mathrm{x}}}\right)=g\left(T_{\mathrm{x}}\right)\left(S_{T_{\mathrm{x}}}-K\right)^{+}
$$


where $g(t)$ denotes the probability that the policy has not been lapsed by the time $t$. Instead of assuming that $g$ is deterministic, as in Section 3.1, we are interested in the case when the decision of lapse or surrender depends on whether the underlying asset price has breached over some pre-specified barrier. As shown in Milevsky and Posner (2001) and Gerber et al. (2012, 2013), the valuation of the GMDB contract under which the decision of lapses or surrenders depends on the crossings of the pre-specified barriers is equivalent to the fair valuation of barrier options. In addition, Dong (2011) also showed that the concept of the barrier option under the jump-diffusion framework can be applied to the fair valuation of some life insurance contracts with the consideration of default risk. In this respect, we discuss the fair valuation of barrier options in this section.

A knock-in barrier option comes into existence if the underlying asset has reached a prespecified barrier or interval before maturity $T_{\mathrm{x}}$, whereas a knock-out option expires worthlessly if the barrier or interval has been reached before maturity $T_{\mathrm{x}}$. The connection between the knock-in and knock-out option can be expressed by the following parity relation:

$$
\text { Knock-in option }+ \text { Knock-out option = basic option. }
$$

In this section, we illustrate the valuation of up-and-in and up-and-out call options in our model. Other barrier options such as up-and-out, down-and-in, down-and-out, and double barrier options follow analogously.

Let $\tau_{U} \triangleq \inf \left\{t \geq 0: S_{t} \geq U\right\}$ and assume that $S_{0}<U$ and $J_{0}=i$. The equity-indexed benefit functions at the death of the investor, $T_{\mathrm{x}}$, for up-and-in and up-and-out option with strike price $K$, respectively, are:

$$
\begin{aligned}
b_{\text {up-and-in }}\left(T_{\mathrm{x}}, S_{T_{\mathrm{x}}}\right) & \triangleq\left(S_{T_{\mathrm{x}}}-K\right)^{+} 1_{\left\{\tau_{U}<T_{\mathrm{x}}\right\}}, \\
b_{\text {up-and-out }}\left(T_{\mathrm{x}}, S_{T_{\mathrm{x}}}\right) & \triangleq\left(S_{T_{\mathrm{x}}}-K\right)^{+} 1_{\left\{\tau_{U}>T_{\mathrm{x}}\right\}} .
\end{aligned}
$$

Denote by $\operatorname{UIC}\left(S_{0}, K, U, T_{\mathrm{x}}, i\right)$ and $\operatorname{UOC}\left(S_{0}, K, U, T_{\mathrm{x}}, i\right)$ the fair values of the up-and-in and up-and-out call options, respectively. In other words,

$$
\begin{aligned}
U I C\left(S_{0}, K, U, T_{\mathrm{x}}, i\right) & =\mathbb{E}_{i}\left[\mathrm{e}^{-r T_{\mathrm{x}}}\left(S_{T_{\mathrm{x}}}-K\right)^{+} 1_{\left\{\tau_{U}<T_{\mathrm{x}}\right\}}\right], \\
\operatorname{UOC}\left(S_{0}, K, U, T_{\mathrm{x}}, i\right) & =\mathbb{E}_{i}\left[\mathrm{e}^{-r T_{\mathrm{x}}}\left(S_{T_{\mathrm{x}}}-K\right)^{+} 1_{\left\{\tau_{U}>T_{\mathrm{x}}\right\}}\right] .
\end{aligned}
$$

In terms of the inverse Laplace transform, their fair values under our model are given as follows.

Theorem 3.6. Let $T_{\mathrm{x}}$ be the time-until-death random variable with the density function (21).

For all $\varphi$ satisfying $0<\varphi<\min _{j}\left\{\eta_{j 1}-1\right\}$ and $\alpha+r>\max _{j \in E}\left\{\kappa_{j}(\varphi+1)\right\}$, where $\kappa_{j}(u)$ is given by (4), the prices of the up-and-in and up-and-out call options take the respective forms:

$$
\begin{aligned}
U I C\left(S_{0}, K, U, T_{\mathrm{x}}, i\right)= & \mathcal{L}_{k}^{-1}\left(\frac{\alpha \chi^{-\varphi} S_{0}^{\varphi+1}}{\varphi(\varphi+1)} \sum_{j, n \in E} \mathbb{E}_{i}\left[\mathrm{e}^{-(\alpha+r) \tau_{U}+(\varphi+1) X_{\tau_{U}}} 1_{\left\{J_{\tau_{U}}=j\right\}}\right] \boldsymbol{A}_{j n} \mathbf{1}\right) \\
\operatorname{UOC}\left(S_{0}, K, U, T_{\mathrm{x}}, i\right)= & \mathcal{L}_{k}^{-1}\left(\frac{\alpha \chi^{-\varphi} S_{0}^{\varphi+1}}{\varphi(\varphi+1)} \mathbf{e}_{i}^{\top}((\alpha+r) \mathbf{I}-\mathbf{K}[\varphi+1])^{-1} \mathbf{1}\right. \\
& -\frac{\alpha \chi^{-\varphi} S_{0}^{\varphi+1}}{\varphi(\varphi+1)} \sum_{j, n \in E} \mathbb{E}_{i}\left[\mathrm{e}^{\left.\left.-(\alpha+r) \tau_{U}+(\varphi+1) X_{\tau_{U}} 1_{\left\{J_{\tau_{U}}=j\right\}}\right] \boldsymbol{A}_{j n} \mathbf{1}\right)}\right.
\end{aligned}
$$


where $k=-\log (K / \chi), \boldsymbol{A} \triangleq((\alpha+r) \mathbf{I}-\boldsymbol{K}[\varphi+1])^{-1}$ with $\boldsymbol{A}_{j n}$ denoting the $n^{\text {th }}$ element of the matrix $\boldsymbol{A}$.

Proof. We first compute the fair value of the up-and-in call option in (33). Under the assumptions, the Laplace transform exists and using Fubini's Theorem, we then have

$$
\begin{aligned}
& \mathcal{L}_{\varphi}\left(U I C\left(S_{0}, K, U, T_{\mathrm{x}}, i\right)\right) \\
& =\int_{-\infty}^{\infty} \mathrm{e}^{-\varphi k} \int_{0}^{\infty} \alpha \mathrm{e}^{-(\alpha+r) T} \mathbb{E}_{i}\left[\chi\left(\frac{S_{T}}{\chi}-\mathrm{e}^{-k}\right)^{+} 1_{\left\{\tau_{U}<T\right\}}\right] \mathrm{d} T \mathrm{~d} k \\
& =\frac{\alpha \chi}{\varphi(\varphi+1)}\left(\frac{S_{0}}{\chi}\right)^{\varphi+1} \mathbb{E}_{i}\left[\int_{\tau_{U}}^{\infty} \sum_{n \in E} \mathrm{e}^{-(\alpha+r) T} \mathrm{e}^{(\varphi+1) X_{T}} 1_{\left\{J_{T}=n\right\}} \mathrm{d} T\right] \\
& =\frac{\alpha \chi}{\varphi(\varphi+1)}\left(\frac{S_{0}}{\chi}\right)^{\varphi+1} \mathbb{E}_{i}\left[\int_{0}^{\infty} \sum_{n \in E} \mathrm{e}^{-(\alpha+r)\left(s+\tau_{U}\right)} \mathrm{e}^{(\varphi+1) X_{s+\tau_{U}}} 1_{\left\{J_{s+\tau_{U}}=n\right\}} \mathrm{d} s\right] \\
& =\frac{\alpha \chi}{\varphi(\varphi+1)}\left(\frac{S_{0}}{\chi}\right)^{\varphi+1} \mathbb{E}_{i}\left[\sum_{j \in E} \mathrm{e}^{-(\alpha+r)\left(\tau_{U}\right)} \mathrm{e}^{(\varphi+1) X_{\tau_{U}}} 1_{\left\{J_{\tau_{U}}=j\right\}}\right. \\
& \left.\times \sum_{n \in E} \mathbb{E}_{j}\left[\int_{0}^{\infty} \mathrm{e}^{-(\alpha+r) s+(\varphi+1)\left(X_{s+\tau_{U}}-X_{\tau_{U}}\right)} 1_{\left\{J_{s+\tau_{U}}=n\right\}} \mathrm{d} s \mid \mathcal{F}_{\tau_{U}}\right]\right] \\
& =\frac{\alpha \chi}{\varphi(\varphi+1)}\left(\frac{S_{0}}{\chi}\right)^{\varphi+1} \sum_{j \in E} \mathbb{E}_{i}\left[\mathrm{e}^{-(\alpha+r) \tau_{U}} \mathrm{e}^{(\varphi+1) X_{\tau_{U}}} 1_{\left\{J_{\tau_{U}}=j\right\}} \int_{0}^{\infty} \sum_{n \in E}\left(\mathrm{e}^{-(\alpha+r) \mathbf{I} s+s \mathbf{K}[\varphi+1]}\right)_{j n} \mathrm{~d} s\right] \text {, }
\end{aligned}
$$

where the third equality follows from the independence assumption of $T_{\mathrm{x}}$ and $\left(S_{t}\right)_{t \geq 0}$, and the last equality follows from the strong Markov property of $X_{t}$. The result follows by invoking the invertibility of the matrix $((\alpha+r) \mathbf{I}-\mathbf{K}[\varphi+1])$ (see Lemma 2.5). The corresponding up-and-out call option in (34) now follows by combining the price of the up-and-in option in (34), the basic call option in (24), and the parity relation in (32).

To carry on with the remaining computations, we need to know how to compute the term $\mathbb{E}_{i}\left[\mathrm{e}^{-(\alpha+r) \tau_{U}} \mathrm{e}^{(\varphi+1) X_{\tau_{U}}} 1_{\left\{J_{\tau_{U}}=j\right\}}\right]$, which is covered in Section A.3.

\subsection{Dynamic fund protection}

Dynamic fund protection provides a guarantee that the account value of the investor never drops below a pre-specified level throughout the life of the contract. More specifically, let $S_{t}$ be the asset price at time $t$ with $0<L<S_{0}$. Denote by $n_{t}$ the number of units of $S_{t}$ purchased at time $t$. In a dynamic fund protection, as soon as the investor's account value drops below the guaranteed level $L, n_{t}$ units of $S_{t}$ will automatically be credited into his account to restore the account value to the guaranteed level $L$.

Following the discussion in Gerber et al. (2012), $n_{t}$ admits the following representation:

$$
n_{t}=\max \left(1, \max _{0 \leq s \leq t} \frac{L}{S_{s}}\right)
$$


which indicates that the equity-indexed benefit function for the dynamic fund protection at the death of the investor $T_{\mathrm{x}}$ is

$$
b_{\text {Protection }}\left(T_{\mathrm{x}}, S_{T_{\mathrm{x}}}\right) \triangleq \max \left(1, \max _{0 \leq t \leq T_{\mathrm{x}}} \frac{L}{S_{t}}\right) S_{T_{\mathrm{x}}} .
$$

Since

$$
\mathbb{E}_{i}\left[\mathrm{e}^{-r T_{\mathrm{x}}} \max \left(1, \max _{0 \leq t \leq T_{\mathrm{x}}} \frac{L}{S_{t}}\right) S_{T_{\mathrm{x}}}\right]=\mathbb{E}_{i}\left[\mathrm{e}^{-r T_{\mathrm{x}}} S_{T_{\mathrm{x}}}\left(\max _{0 \leq t \leq T_{\mathrm{x}}} \frac{L}{S_{t}}-1\right)^{+}\right]+S_{0},
$$

we evaluate the expectation above by considering $S_{T_{\mathrm{x}}}$ as the numéraire.

Let $\tilde{\mathbb{Q}}$ be the equivalent martingale measure defined in (10). Under measure $\tilde{\mathbb{Q}}$, we have

$$
S_{0} \mathbb{E}_{i}\left[\mathrm{e}^{-r T_{\mathrm{x}}} \frac{S_{T_{\mathrm{x}}}}{S_{0}}\left(\max _{0 \leq t \leq T_{\mathrm{x}}} \frac{L}{S_{t}}-1\right)^{+}\right]=S_{0} \tilde{\mathbb{E}}_{i}\left[\left(\max _{0 \leq t \leq T_{\mathrm{x}}} \frac{L}{S_{t}}-1\right)^{+}\right] .
$$

By invoking Proposition 2.6, the remaining computation follows in line with the case of computing standard lookback options under measure $\tilde{\mathbb{Q}}$ in Section 3.2.

\subsection{Dynamic withdrawal benefits}

In Section 3.4, we focus on the case when the investor enters the dynamic fund protection with an intention that his fund or asset price never drops below a pre-specified level. In this section, we consider the case when the investor is interested in entering a contract such that he will be paid "dividends" whenever the fund or the asset price reaches above a prespecified level. Following the nomenclature used in Ko et al. (2010), such a contract is called a dynamic withdrawal benefit.

More specifically, let $S_{t}$ be the asset price at time $t$ and $L$ be the level of the "dividend barrier" with $L \geq S_{0}$. Whenever the investor's account value reaches above $L$ within the duration of the dynamic withdrawal benefit, just enough units of $S_{t}$, denoted by $n_{t}$, will be sold so that his account value stays at level $L$.

Following the same reasoning as in Section 3.4, we have

$$
n_{t}=\min \left(1, \min _{0 \leq s \leq t} \frac{L}{S_{s}}\right) .
$$

The dynamic withdrawal benefit is a contract in which the equity-indexed benefit function at the death of the investor, $T_{\mathrm{x}}$, is of the form

$$
b_{\text {withdrawal }}\left(T_{\mathrm{x}}, S_{T_{\mathrm{x}}}\right) \triangleq 1-\min \left(1, \min _{0 \leq t \leq T_{\mathrm{x}}} \frac{L}{S_{t}}\right) S_{T_{\mathrm{x}}} .
$$


The discounted value of the dynamic withdrawal benefit at time 0 is given by:

$$
\begin{aligned}
\mathbb{E}\left[\mathrm{e}^{-r T_{\mathrm{x}}}\left(1-\min \left(1, \min _{0 \leq t \leq T_{\mathrm{x}}} \frac{L}{S_{t}}\right)\right) S_{T_{\mathrm{x}}}\right] & =\mathbb{E}\left[\mathrm{e}^{-r T_{\mathrm{x}}} S_{T_{\mathrm{x}}}\left(1-\min _{0 \leq t \leq T_{\mathrm{x}}} \frac{L}{S_{t}}\right)^{+}\right] \\
& =S_{0} \mathbb{E}\left[\mathrm{e}^{-r T_{\mathrm{x}}} \frac{S_{T_{\mathrm{x}}}}{S_{0}}\left(1-\min _{0 \leq t \leq T_{\mathrm{x}}} \frac{L}{S_{t}}\right)^{+}\right] \\
& =S_{0} \tilde{\mathbb{E}}\left[\left(1-\min _{0 \leq t \leq T_{\mathrm{x}}} \frac{L}{S_{t}}\right)^{+}\right]
\end{aligned}
$$

where $\tilde{\mathbb{Q}}$ is the equivalent martingale measure defined in (10). By invoking Proposition 2.6, the remaining computation follows analogously as in the case of the dynamic fund protection.

\section{T-year contingent options and De Moivre's Law}

\subsection{T-year contingent options}

In Section 3, we assume that the maturities of the contracts coincide with the death of the investor. In this section, we relax this assumption by working with the defective probability function, denoted by $\tilde{f}_{T_{\mathrm{x}}}$ (see Gerber et al. (2012)), where

$$
\tilde{f}_{T_{\mathrm{x}}}(t) \triangleq f_{T_{\mathrm{x}}}(t) 1_{\left\{T_{\mathrm{x}}<T\right\}}
$$

In other words, the GMDB contracts only provide the payment if the death of the investor comes before the pre-specified time horizon $T$.

Instead of reworking every problem in Section 3 under $\tilde{f}_{T_{\mathrm{x}}}$, we illustrate the flexibility of the Laplace transform technique by valuing a basic call option from Section 3.1 and the standard lookback option from Section 3.2.1 under the defective probability function $\tilde{f}_{T_{\mathrm{x}}}$.

For the following examples, let $T_{\mathrm{x}}$ be the time-until-death random variable with the density function (37).

Example 4.1. Following the proof of Theorem 3.1, the Laplace transform of the T-year maturity basic call option, with respect to $k=-\log (K / \chi)$ where $\chi>K$, is given by

$$
\begin{aligned}
& \int_{-\infty}^{\infty} \mathrm{e}^{-\varphi k} \chi \mathbb{E}_{i}\left[\mathrm{e}^{-r T_{\mathrm{x}}}\left(\frac{S_{0}}{\chi} \mathrm{e}^{X_{T_{\mathrm{x}}}}-\mathrm{e}^{-k}\right)^{+}\right] \mathrm{d} k \\
= & \int_{0}^{\infty} \alpha \mathrm{e}^{-(\alpha+r) t} 1_{\{t<T\}} \chi \mathbb{E}_{i}\left[\int_{-\log \left(\frac{S_{0}}{\chi} \mathrm{e}^{X_{t}}\right)}^{\infty} \mathrm{e}^{-\varphi k}\left(\frac{S_{0}}{\chi} \mathrm{e}^{X_{t}}-\mathrm{e}^{-k}\right) \mathrm{d} k\right] \mathrm{d} t \\
= & \chi\left(\frac{S_{0}}{\chi}\right)^{\varphi+1} \frac{\alpha}{\varphi(\varphi+1)} \mathbf{e}_{i}^{\top}\left[\mathbf{I}-\mathrm{e}^{-((\alpha+r) \mathbf{I}-\mathbf{K}[\varphi+1]) T}\right]((\alpha+r) \mathbf{I}-\mathbf{K}[\varphi+1])^{-1} \mathbf{1} .
\end{aligned}
$$


Example 4.2. Consider the valuation of the floating-strike lookback put option with maturity T. Following the proof of Theorem 3.2, we readily have

$$
\begin{aligned}
L P\left(S_{0}, M, T_{\mathrm{x}}, i\right)= & S_{0} \int_{z}^{\infty} \mathrm{e}^{m} \int_{0}^{\infty} \alpha \mathrm{e}^{-(\alpha+r) t} 1_{\{t<T\}} \mathbb{Q}_{i}\left[M_{t}^{X} \geq m\right] \mathrm{d} t \mathrm{~d} m \\
& +M \int_{0}^{\infty} \mathrm{e}^{-(\alpha+r) t} 1_{\{t<T\}} \mathrm{d} t-S_{0}
\end{aligned}
$$

Since

$$
\int_{0}^{\infty} \mathrm{e}^{-(\alpha+r) t} 1_{\{t<T\}} \mathbb{Q}_{i}\left[\tau_{m}<t\right] \mathrm{d} t=\frac{1}{\alpha+r}\left[\sum_{j \in E} \mathbb{E}_{i}\left[\mathrm{e}^{-(\alpha+r) \tau_{m}} 1_{\left\{J_{\tau_{m}}=j\right\}}\right]-\mathrm{e}^{-(\alpha+r) T}\right],
$$

we have the value of the T-year standard floating-strike lookback put as follows

$$
\begin{aligned}
L P\left(S_{0}, M, T_{\mathrm{x}}, i\right)= & \frac{\alpha S_{0}}{\alpha+r} \int_{z}^{\infty} \mathrm{e}^{m}\left[\sum_{j \in E} \mathbb{E}_{i}\left[\mathrm{e}^{-(\alpha+r) \tau_{m}} 1_{\left\{J_{\tau_{m}}=j\right\}}\right]-\mathrm{e}^{-(\alpha+r) T}\right] \mathrm{d} m \\
& +\frac{M \alpha}{\alpha+r}\left(1-\mathrm{e}^{-(\alpha+r) T}\right)-S_{0}
\end{aligned}
$$

As shown in Examples 4.1 and 4.2, the valuations of $T$-year options come effortlessly under the Laplace transform framework.

\subsection{De Moivre's Law}

The explicit formulation of the $T$-year option can be extended to the case when the timeuntil-death random variable $T_{\mathrm{x}}$ is uniformly distributed. More specifically, from Gerber et al. (2012), consider the case when $T_{\mathrm{x}}$ has a uniform distribution on $[0, \omega-x]$, where $\omega$ is the maximal possible age. Consider the maturity of the contract $T \leq \omega-x$. It follows by De Moivre's Law that the truncated density function of $T_{\mathrm{x}}$ under the uniform distribution on $[0, \omega-x]$, denoted by $f_{T_{\mathrm{x}}}^{\text {unif }}$, takes the form

$$
f_{T_{\mathrm{x}}}^{u n i f}(t) \triangleq \frac{1}{\omega-x} \lim _{\alpha \rightarrow 0} \frac{1}{\alpha} f_{T_{\mathrm{x}}}(t) 1_{\{t<T\}} .
$$

As discussed in Gerber et al. (2012), the seemingly innocuous expression (38) has a profound consequence: the valuation scheme we have discussed so far under the exponential mortality density function can be carried out in an exact manner by simply replacing (21) with (38). The following example illustrates this insight in greater detail.

Example 4.3. Let us illustrate the idea by revisiting the $T$-maturity basic call option in Example 4.1 under $f_{T_{\mathrm{x}}}^{\text {unif }}$. For $\varphi \in\left(0, \min \left\{\eta_{j 1}-1\right\}\right)$ and $k=-\log (K / \chi)$, an application of 
Fubini's Theorem gives

$$
\begin{aligned}
& \int_{-\infty}^{\infty} \mathrm{e}^{-\varphi k} \chi \mathbb{E}_{i}\left[\mathrm{e}^{-r T_{\mathrm{x}}}\left(\frac{S_{0}}{\chi} \mathrm{e}^{X_{T_{\mathrm{x}}}}-\mathrm{e}^{-k}\right)^{+}\right] \mathrm{d} k \\
= & \int_{0}^{\infty} \mathrm{e}^{-r t} f_{T_{\mathrm{x}}}^{u n i f}(t) \chi \mathbb{E}_{i}\left[\int_{-\log \left(\frac{S_{0}}{\chi} \mathrm{e}^{X_{t}}\right)}^{\infty} \mathrm{e}^{-\varphi k}\left(\frac{S_{0}}{\chi} \mathrm{e}^{X_{t}}-\mathrm{e}^{-k}\right) \mathrm{d} k\right] \mathrm{d} t \\
= & \lim _{\alpha \rightarrow 0} \int_{0}^{\infty} \alpha \mathrm{e}^{-(\alpha+r) t} \frac{1}{\alpha(\omega-x)} 1_{\{t<T\}} \chi \mathbb{E}_{i}\left[\int_{-\log \left(\frac{S_{0}}{\chi} \mathrm{e}^{X_{t}}\right)}^{\infty} \mathrm{e}^{-\varphi k}\left(\frac{S_{0}}{\chi} \mathrm{e}^{X_{t}}-\mathrm{e}^{-k}\right) \mathrm{d} k\right] \mathrm{d} t \\
= & \frac{\chi\left(\frac{S_{0}}{\chi}\right)^{\varphi+1}}{\varphi(\varphi+1)(\omega-x)} \mathbf{e}_{i}^{\top}\left[\mathbf{I}-\mathrm{e}^{-(r \mathbf{I}-\mathbf{K}[\varphi+1]) T}\right](r \mathbf{I}-\mathbf{K}[\varphi+1])^{-1} \mathbf{1} .
\end{aligned}
$$

Other options considered in this paper can analogously be computed under the uniformly mortality density function $f_{T_{\mathrm{x}}}^{u n i f}$.

\section{$5 \quad$ Numerical examples}

\subsection{GMDBs under a life table}

\subsubsection{Calibrating combinations of exponential functions to a life table}

To extend the scope of the Laplace transform framework beyond the case of exponentiallydistributed $T_{\mathrm{x}}$, we price the life-contingent claims discussed in Section 3 based on a life table in this section. More specifically, we revisit the valuations of the basic options, lookback options, and the barrier options by calibrating combinations of exponential functions to a life table. We use the Illustrative Life Table in Appendix 2A of Bowers et al. (1997) as our mortality table. Let $f(t, l ; \mathbf{a}, \mathbf{b})$ be a combination of exponential functions, i.e.

$$
f\left(t, l ; \mathbf{a}_{l}, \mathbf{b}_{l}\right) \triangleq \sum_{i=1}^{l} \mathrm{a}_{i} \mathrm{e}^{-\mathrm{b}_{i} t}
$$

where $\mathbf{a}_{l} \triangleq\left(\mathrm{a}_{1}, \ldots, \mathrm{a}_{l}\right), \mathrm{b}_{l} \triangleq\left(\mathrm{b}_{1}, \ldots, \mathrm{b}_{l}\right)$ with $\mathrm{b}_{i}>0$, for $i=1, \ldots, l$, and $\sum_{i=1}^{l} \mathrm{a}_{i}=1$. We denote by $\mathcal{M}_{l}$ the mortality model with the survival function $f\left(t, l ; \mathbf{a}_{l}, \mathbf{b}_{l}\right)$ and we denote $T_{\mathrm{x}}^{\mathcal{M}_{l}}$ for the corresponding time-until-death random variable under the mortality model $\mathcal{M}_{l}$.

We first fit the mortality data for a person at age $\mathrm{x}$ with the survival function $f\left(t, l ; \mathbf{a}_{l}, \mathbf{b}_{l}\right)$ in (39). More specifically, for fixed $l, n \in \mathbb{N}$, we find $\left(\mathbf{a}_{l}, \mathbf{b}_{l}\right)$ by solving the following non-linear least-squares problem:

$$
\mathcal{S}\left(\mathbf{a}_{l}, \mathbf{b}_{l}\right) \triangleq \min _{\mathbf{a}_{l}, \mathbf{b}_{l}} \sum_{t=1}^{n}\left[{ }_{t} p_{\mathbf{x}}-f\left(t, l ; \mathbf{a}_{l}, \mathbf{b}_{l}\right)\right]^{2},
$$

where ${ }_{t} p_{\mathrm{x}}$ denotes the empirical probability that a person at age $\mathrm{x}$ will survive for $t$ more years. We denote by $q_{\mathrm{x}+t}$ and $\hat{q}_{\mathrm{x}+t}^{l}$, respectively, the one-year mortality rate of a person at age 
$\mathrm{x}$, in $t$ years based on the life table and based on the calibrated survival function $f\left(t, l ; \mathbf{a}_{l}, \mathbf{b}_{l}\right)$, i.e.

$$
\hat{q}_{\mathbf{x}+t}^{l} \triangleq \frac{f\left(t, l ; \mathbf{a}_{l}, \mathbf{b}_{l}\right)-f\left(t+1, l ; \mathbf{a}_{l}, \mathbf{b}_{l}\right)}{f\left(t, l ; \mathbf{a}_{l}, \mathbf{b}_{l}\right)}
$$

For illustration, we calibrate three survival functions $f\left(t, l ; \mathbf{a}_{l}, \mathbf{b}_{l}\right)$, for $l=3,5,10$, using the Illustrative Life Table for a person at age $\mathrm{x}=30$ when $n=25$. The fitted combinations of exponential functions are shown as follows:

$$
\begin{aligned}
f\left(t, 3 ; \mathbf{a}_{3}, \mathbf{b}_{3}\right)= & -1.6862 \mathrm{e}^{-0.0387858 t}+0.1623 \mathrm{e}^{-0.109792 t}+2.5239 \mathrm{e}^{-0.0197795}, \\
f\left(t, 5 ; \mathbf{a}_{5} . \mathbf{b}_{5}\right)= & 1.0893 \mathrm{e}^{-0.0212392 t}+0.8730 \mathrm{e}^{-0.0219805 t}+1.0817 \mathrm{e}^{-0.0708229} \\
& +0.6860 \mathrm{e}^{-0.0215123 t}-2.7301 \mathrm{e}^{-0.0481505 t}, \\
f\left(t, 10 ; \mathbf{a}_{10}, \mathbf{b}_{10}\right)= & -0.7306 \mathrm{e}^{-0.088166 t}+0.2259 \mathrm{e}^{-0.179319 t}+0.6499 \mathrm{e}^{-0.0142838} \\
& +0.3749 \mathrm{e}^{-0.172757 t}+0.4144 \mathrm{e}^{-0.172873 t}-0.3345 \mathrm{e}^{-0.0904136 t} \\
& +0.4155 \mathrm{e}^{-0.0158551 t}-0.5267 \mathrm{e}^{-0.270833 t}+0.4198 \mathrm{e}^{-0.0164353 t} \\
& +0.0914 \mathrm{e}^{-0.437897 t},
\end{aligned}
$$

with total errors $\sum_{t=1}^{n}\left[{ }_{t} p_{30}-f\left(t, l ; \mathbf{a}_{l}, \mathbf{b}_{l}\right)\right]^{2}$ equal to 0.0000159489 for $l=3,0.0000125984$ for $l=5$, and 0.00000188246 for $l=10$.

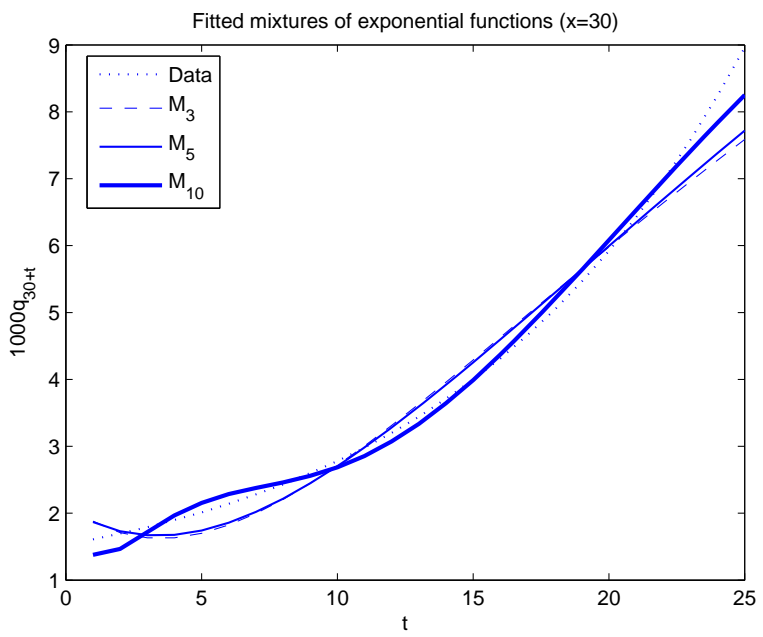

Figure 1: Performance of the fitted combinations of exponential functions $f\left(t, l ; \mathbf{a}_{l}, \mathbf{b}_{l}\right)$ for $l=3,5,10$. Here, $M_{l}$ corresponds to $\hat{q}_{30+t}^{l}$ computed under the mortality model $\mathcal{M}_{l}$ (see (40)), for $l=3,5,10$.

Figure 1 provides the visual comparisons of the computed mortality rates $\hat{q}_{30+t}^{3}, \hat{q}_{30+t}^{5}$, and $\hat{q}_{30+t}^{10}$ against the empirical mortality rates $q_{30+t}$.

\subsubsection{Valuations with the calibrated mortality models}

With the calibrated mortality models (Models $\mathcal{M}_{3}, \mathcal{M}_{5}$, and $\mathcal{M}_{10}$ ) in place, we proceed to compute the options discussed in Section 3 under these calibrated mortality models. In 


\begin{tabular}{|c|c|c|c|c|c|}
\hline \multicolumn{7}{|c|}{ Regime 1} \\
\hline$\sigma_{1}$ & $\eta_{11}$ & $\eta_{12}$ & $p_{1}$ & $\lambda_{1}$ & $q_{12}$ \\
\hline 0.1 & 40 & 60 & 0.75 & 2 & 0.1 \\
\hline \multicolumn{7}{|c|}{ Regime 2} \\
\hline$\sigma_{2}$ & $\eta_{21}$ & $\eta_{22}$ & $p_{2}$ & $\lambda_{2}$ & $q_{21}$ \\
\hline 0.4 & 60 & 10 & 0.25 & 0.5 & 0.2 \\
\hline
\end{tabular}

Table 1: Model parameters

particular, we focus on computing the fair values of the basic options, floating strike options, and the up-and-out call options. ${ }^{5}$ For the sake of illustration, we focus on the case of two regimes, i.e. $E=\{1,2\}$. All computations are performed on a desktop with an Intel ${ }^{\circledR} 1.4 \mathrm{GHz}$ processor.

Table 1 summarizes the model parameters for the fair valuations of the options in this section. In Table 1, Regime 1 represents the "good" economy case, i.e. when the underlying asset has low volatility $\left(\sigma_{1}\right)$ and higher probability $\left(p_{1}\right)$ of experiencing small-sized upward $\left(\eta_{11}\right)$ jumps than experiencing small-sized downward $\left(\eta_{12}\right)$ jumps. On the other hand, Regime 2 represents the "bad economy" case, i.e. when the underlying asset has high volatility $\left(\sigma_{2}\right)$ and lower probability $\left(p_{2}\right)$ of experiencing small-sized upward $\left(\eta_{21}\right)$ jumps than experiencing larger-sized downward $\left(\eta_{22}\right)$ jumps. Moreover, we set $q_{12}=0.1$ and $q_{21}=0.2$ implying that the average durations of the good and bad economic states are $\frac{1}{q_{12}}=10$ years and $\frac{1}{q_{21}}=5$ years, respectively.

Table 2 displays prices of the basic put option with various strike prices. The average computation time for each option is 0.0624 seconds. In Table 2, we see that increasing the strike price $K$ increases the fair values of the corresponding basic put option. This is logical as increasing the strike price increases the probability of the put option to be in-the-money at time $T_{\mathrm{x}}$.

Applying Theorem 3.2, we obtain values of floating strike lookback put options. The numerical results are presented in Table 3. As discussed in Section 3.2, Theorem 3.2 yields a closed-form solution for each lookback put option and hence the computational time is negligible.

We now turn to numerical examples for up-and-out call options. We perform the bilateral Abate-Whitt algorithm to Theorem 3.3 to recover the value of the up-and-out call option. The results are summarized in Table 4. The average computation time is 0.2340 seconds. In Table 4, we see that increasing the upper barrier $H$ increases the corresponding price of the up-and-out call option. This can be explained by the fact that increasing the upper barrier reduces the up-crossing probability of the underlying asset. This in turn increases the probability of the up-and-out option remaining "alive" at maturity $T_{\mathrm{x}}$, thereby increasing the value of the corresponding option.

Next, we compare the option values in the good (Regime 1) and bad (Regime 2) economic regimes in Tables 2, 3, and 4, based on the total squared volatility of the asset price in each

\footnotetext{
${ }^{5}$ In Section 3, we have shown that the dynamic fund protection and dynamic withdrawal benefit are structurally equivalent to lookback options. In this respect, it suffices for us to compute the fair values of lookback options for illustration here.
} 


\begin{tabular}{|c|c|c|c|c|c|c|c|}
\hline \multicolumn{4}{|c|}{ Initial regime: Regime $1\left(J_{0}=1\right)$} & \multicolumn{4}{|c|}{ Initial regime: Regime $2\left(J_{0}=2\right)$} \\
\hline $\mathrm{K}^{\mathcal{M}_{l}}$ & $\mathcal{M}_{3}$ & $\mathcal{M}_{5}$ & $\mathcal{M}_{10}$ & & $\mathcal{M}_{3}$ & $\mathcal{M}_{5}$ & $\mathcal{M}_{10}$ \\
\hline 95 & 0.6060 & 0.6402 & 0.5912 & 95 & 1.4024 & 1.4506 & 1.4301 \\
\hline 100 & 0.7519 & 0.7876 & 0.7377 & 100 & 1.5800 & 1.6309 & 1.6099 \\
\hline 105 & 0.9073 & 0.9445 & 0.8940 & 105 & 1.7657 & 1.8193 & 1.7982 \\
\hline 110 & 1.0714 & 1.1105 & 1.0596 & 110 & 1.9589 & 2.0155 & 1.9944 \\
\hline 115 & 1.2439 & 1.2849 & 1.2339 & 115 & 2.1593 & 2.2190 & 2.1982 \\
\hline 120 & 1.4241 & 1.4672 & 1.4163 & 120 & 2.3665 & 2.4294 & 2.4089 \\
\hline 125 & 1.6117 & 1.6571 & 1.6065 & 125 & 2.5800 & 2.6462 & 2.6263 \\
\hline
\end{tabular}

Table 2: Basic put option with various strike prices $K$ and mortality models $\mathcal{M}_{d}$, for $d=$ $3,5,10$.

Note: The computations of the basic put options are performed based on the parameter inputs given in Table 1. Other parameters are $S_{0}=100, r=0.05, N=50, m=18$, and $\chi=10^{30}$. During the implementation of the bilateral Abate-Whitt algorithm, the scaling parameter $\chi$ is set to ensure that $\alpha+r>\max _{j \in E}\left\{\kappa_{j}(\varphi+1)\right\}$ in Theorem 3.1 is satisfied.

\begin{tabular}{|c|c|c|c|}
\hline \multicolumn{4}{|c|}{ Initial regime: Regime $1\left(J_{0}=1\right)$} \\
\hline $\mathcal{M}_{l}$ & $\mathcal{M}_{3}$ & $\mathcal{M}_{5}$ & $\mathcal{M}_{10}$ \\
\hline 105 & 60.7740 & 60.7333 & 60.7136 \\
110 & 60.7908 & 60.7500 & 60.7293 \\
115 & 60.8174 & 60.7765 & 60.7543 \\
120 & 60.8532 & 60.8123 & 60.7883 \\
125 & 60.8978 & 60.8570 & 60.8310 \\
130 & 60.9508 & 60.9102 & 60.8822 \\
135 & 61.0119 & 60.9716 & 60.9417 \\
\hline
\end{tabular}

\begin{tabular}{|c|c|c|c|}
\hline \multicolumn{4}{|c|}{ Initial regime: Regime $2\left(J_{0}=2\right)$} \\
\hline $\mathcal{M}_{l}$ & $\mathcal{M}_{3}$ & $\mathcal{M}_{5}$ & $\mathcal{M}_{10}$ \\
\hline 105 & 62.6244 & 62.6127 & 62.6229 \\
\hline 110 & 62.6462 & 62.6346 & 62.6448 \\
\hline 115 & 62.6810 & 62.6698 & 62.6798 \\
\hline 120 & 62.7279 & 62.7172 & 62.7270 \\
\hline 125 & 62.7861 & 62.7759 & 62.7855 \\
\hline 130 & 62.8547 & 62.8453 & 62.8547 \\
\hline 135 & 62.9331 & 62.9246 & 62.9338 \\
\hline
\end{tabular}

Table 3: Floating-strike lookback put option with various initial maximum asset prices $M$ and mortality models $\mathcal{M}_{l}$, for $d=3,5,10$.

Note: The computations of the floating-strike lookback put options are performed based on the parameter inputs given in Table 1. Other parameters are $S_{0}=100$ and $r=0.05$. 


\begin{tabular}{|c|c|c|c||c|c|c|c|}
\hline \multicolumn{6}{|c|}{ Initial regime: Regime $1\left(J_{0}=1\right), \mathrm{K}=95$} & \multicolumn{3}{|c|}{ Initial regime: Regime $1\left(J_{0}=2\right), \mathrm{K}=95$} \\
\hline $\mathcal{M}_{l}$ & $\mathcal{M}_{3}$ & $\mathcal{M}_{5}$ & $\mathcal{M}_{10}$ & $\mathcal{M}_{l}$ & $\mathcal{M}_{3}$ & $\mathcal{M}_{5}$ & $\mathcal{M}_{10}$ \\
\hline 115 & 0.1246 & 0.1262 & 0.1160 & 115 & 0.0814 & 0.0835 & 0.0828 \\
120 & 0.1600 & 0.1618 & 0.1495 & 120 & 0.1061 & 0.1082 & 0.1069 \\
125 & 0.1950 & 0.1971 & 0.1837 & 125 & 0.1317 & 0.1339 & 0.1319 \\
130 & 0.2299 & 0.2323 & 0.2187 & 130 & 0.1581 & 0.1604 & 0.1580 \\
135 & 0.2650 & 0.2678 & 0.2546 & 135 & 0.1853 & 0.1878 & 0.1849 \\
140 & 0.3004 & 0.3036 & 0.2914 & 140 & 0.2133 & 0.2160 & 0.2128 \\
145 & 0.3363 & 0.3399 & 0.3291 & 145 & 0.2420 & 0.2450 & 0.2415 \\
\hline
\end{tabular}

Table 4: Up-and-out call option with various upward barriers $H$ and mortality models $\mathcal{M}_{d}$, for $d=3,5,10$.

Note: The computations of the up-and-out call options are performed based on the parameter inputs given in Table 1. Other parameters are $S_{0}=100, r=0.05, N=50, m=18$, and $\chi=10^{30}$. During the implementation of the bilateral Abate-Whitt algorithm, the scaling parameter $\chi$ is set to ensure that $\alpha+r>$ $\max _{j \in E}\left\{\kappa_{j}(\varphi+1)\right\}$ in Theorem 3.6 is satisfied.

initial regime. ${ }^{6}$ The total squared volatility in Regime 1 is $\sigma_{1, \text { Total }}^{2}=1.1831 \%$, whereas the total volatilty in Regime 2 is $\sigma_{2 \text {,Total }}^{2}=16.3710 \%$. Table 2 confirms our intuition that the prices of basic put options in Regime 2 are higher than those in Regime 1, as higher volatility has an upward price pressure on the values of the corresponding basic options. Analogous patterns and interpretations can be used to explain the fair values of the standard lookback options in Regimes 1 and 2 in Table 3. Yet, the values of the up-and-out call options in Table 4 indicate that the values in Regime 2 are smaller than those in Regime 1 . Since the total volatility in Regime 2 is higher than that in Regime 1, the probability of the underlying asset up-crossing the upper barrier in initial Regime 2 is higher than that in Regime 1, ceteris paribus. Hence, the probability of the up-and-out call option to expire worthless at $T_{\mathrm{x}}$ in Regime 2 is greater than that in Regime 1, which translates into the fair values of the up-and-out call option in Regime 2 to be smaller than those in Regime 1.

Finally, it is worth noting that the fair values in Tables 2,3 , and 4 under $\mathcal{M}_{3}, \mathcal{M}_{5}$, and $\mathcal{M}_{10}$ are close to one another, with the average differences being within one decimal point. From these numerical results, we see that the Laplace transform technique is relatively stable across different (calibrated) mortality models.

${ }^{6}$ The total squared volatility under each initial regime $i$ is given by the following formula:

$$
\sigma_{i, \text { Total }}^{2} \triangleq \sigma_{i}^{2}+\sigma_{i, \mathrm{Jump}}^{2}
$$

where

$$
\sigma_{i, \mathrm{Jump}}^{2} \triangleq \lambda_{i}\left\{\left[\frac{p_{i} \eta_{i 1}}{\eta_{i 1}-2}+\frac{\left(1-p_{i}\right) \eta_{i 2}}{\eta_{i 2}+2}\right]-\left[\frac{p_{i} \eta_{i 1}}{\eta_{i 1}-1}+\frac{\left(1-p_{i}\right) \eta_{i 2}}{\eta_{i 2}+1}\right]^{2}\right\}, \eta_{i 1}>2, i \in E
$$




\section{Conclusion}

In this paper, we have extended the models in Gerber et al. (2012, 2013) to a regimeswitching jump-diffusion model. Using the Laplace transform approach, common options such as basic, lookback, and barrier options, as well as dynamic fund protections, admit closedform representations, thanks to the explicit results provided in Section 2.2. The numerical Laplace inversion scheme enables us to efficiently recover their fair values within a fraction of a second by using any common desktop computer. The advantage of the Laplace transform becomes apparent in the cases of valuing the options with pre-specified maturities. We show that valuing the $T$-year options under the uniform mortality density function comes effortlessly by using the Laplace transform framework. Numerical examples in Section 5.1.2 indicate that our Laplace transform methodology is robust when we approximate the life table with a combination of exponential distributions. One possible extension of our approach is to value GMDBs beyond the family of exponential mortality models. In particular, Lin and Liu (2007) demonstrated that phased-type distributions can also fit life tables accurately. In this respect, it will be interesting to see if the present framework can be adopted to include their phased-type mortality law.

One typical option we have not covered in this paper is the multi-asset option. For the case of a Margrabe option, also known as exchange option, the technique covered in this papers comes naturally with appropriate change of measure. In the case of spread options, in particular in the case of spread call option with payoff structure at maturity: $\left(S_{T_{\mathrm{x}}}^{1}-S_{T_{\mathrm{x}}}^{2}-K, 0\right)^{+}$, special care must be taken as the change of measure technique does not yield a simpler computation. For other options such as dynamic fund protection/withdrawal in which the first passage time distribution plays a critical role in the computation, the problem becomes more delicate as we need to specify the correlation structure of two or more assets. For the case of regime-switching Brownian motions, the problem is manageable as the correlation matrix fully captures the dependence among the assets. With the presence of jumps, the degree of difficulty escalates. The problem of valuing multi-asset options will be left for future research.

\section{Acknowledgements}

The authors appreciate the editor and two anonymous referees for the detailed comments. Chi Chung Siu acknowledges financial support from the Quantitative Finance Research Center (QFRC) at the University of Technology, Sydney. Phillip Yam acknowledges financial support from The Hong Kong RGC GRF 404012 with the project title: Advanced Topics In Multivariate Risk Management In Finance And Insurance, The Chinese University of Hong Kong Direct Grant 2011/2012 Project ID: 2060444. Phillip Yam also expresses his sincere gratitude to the hospitality of both the Hausdorff Center for Mathematics of the University of Bonn and the Mathematisches Forschungsinstitut Oberwolfach (MFO) in the German Black Forest during the preparation of the present work. Hailiang Yang acknowledges support from the Research Grants Council of the Hong Kong Special Administrative Region (project No. HKU 705313P) and a Society of Actuaries' Centers of Actuarial Excellence Research Grant. 


\section{A First passage time distribution of $\left(\tilde{X}_{t}, \tilde{J}_{t}\right)_{t \geq 0}$}

In this appendix, we provide a self-contained exposition on the first passage time distribution of the fluid process $\left(\tilde{X}_{t}, \tilde{J}_{t}\right)_{t \geq 0}$. More importantly, we show the equivalence between the first passage time distribution of the original process $\left(X_{t}, J_{t}\right)_{t \geq 0}$ and that of the fluid process $\left(\tilde{X}_{t}, \tilde{J}_{t}\right)_{t \geq 0}$.

We begin with the conditional independence and memoryless properties of the original process $\left(X_{t}, J_{t}\right)_{t \geq 0}$.

\section{A.1 Conditional independence and memoryless properties of $\left(X_{t}, J_{t}\right)_{t \geq 0}$}

Assume that $X_{0}=0$ and define

$$
\tau_{U} \triangleq \inf \left\{t>0: X_{t} \geq U\right\}, \text { for } U>0 .
$$

Lemma A.1. For any $x>0$, we have

$$
\mathbb{Q}\left(\tau_{U} \leq t, X_{\tau_{U}}-U>x, J_{\tau_{U}}=j\right)=\mathrm{e}^{-\eta_{j 1} x} \mathbb{Q}\left(\tau_{U} \leq t, X_{\tau_{U}}-U>0, J_{\tau_{U}}=j\right) .
$$

Proof. First, note that, for any $x>0$, the event $\left\{X_{\tau_{U}}-U>x, J_{\tau_{U}}=j\right\}$ occurs only by an upward jump. Hence, denoting by $T_{n}, n=1,2, \ldots$, the arrival times of the Poisson process $\left(N_{t}^{j}\right)_{t \geq 0}$, where $J_{T_{n}}=j$, we obtain

$$
\mathbb{Q}\left(\tau_{U} \leq t, X_{\tau_{U}}-U>x, J_{\tau_{U}}=j\right)=\sum_{n=1}^{\infty} \mathbb{Q}\left(T_{n}=\tau_{U} \leq t, X_{\tau_{U}}-U>x, J_{\tau_{U}}=j\right) .
$$

Now, due to the conditional independence and the memoryless property of exponential distributions, we have

$$
\mathbb{Q}\left(X_{T_{n}}-U>x \mid X_{T_{n}-}<U, T_{n}=\tau_{U} \leq t, J_{T_{n}}=j\right)=\mathrm{e}^{-\eta_{j 1} x} .
$$

Since $\mathbb{Q}\left(X_{T_{n}}-U>0 \mid X_{T_{n}-}<U, T_{n}=\tau_{U} \leq t, J_{T_{n}}=j\right)=1$, it follows that

$$
\begin{aligned}
& \mathbb{Q}\left(\max _{0 \leq s<T_{n}} X_{s}<U, X_{T_{n}}-U>x, T_{n}=\tau_{U} \leq t, J_{T_{n}}=j\right) \\
=\mathbb{Q} & \left(X_{T_{n}}-U>x \mid \max _{0 \leq s<T_{n}} X_{s}<U, T_{n}=\tau_{U} \leq t, J_{T_{n}}=j\right) \\
& \quad \times \mathbb{Q}\left(\max _{0 \leq s<T_{n}} X_{s}<U, T_{n}=\tau_{U} \leq t, J_{T_{n}}=j\right) \\
= & \mathrm{e}^{-\eta_{j 1} x} \mathbb{Q}\left(X_{T_{n}}-U>0 \mid \max _{0 \leq s<T_{n}} X_{s}<U, T_{n}=\tau_{U} \leq t, J_{T_{n}}=j\right) \\
& \quad \times \mathbb{Q}\left(\max _{0 \leq s<T_{n}} X_{s}<U, T_{n}=\tau_{U} \leq t, J_{T_{n}}=j\right) \\
= & \mathrm{e}^{-\eta_{j 1} x} \mathbb{Q}\left(\max _{0 \leq s<T_{n}} X_{s}<U, X_{T_{n}}-U>0, T_{n}=\tau_{U} \leq t, J_{T_{n}}=j\right) \\
= & \mathrm{e}^{-\eta_{j 1} x} \mathbb{Q}\left(T_{n}=\tau_{U} \leq t, X_{\tau_{U}}-U>0, J_{\tau_{U}}=j\right) .
\end{aligned}
$$

The lemma follows at once by taking the summation over $n$. 
The next result follows immediately by letting $t \rightarrow \infty$ and observing that, on the event $\left\{X_{\tau_{U}}>U\right\}$, the first passage time $\tau_{U}$ is finite almost surely by definition. That is, we obtain the conditional memoryless property of the exponential jumps.

Corollary A.2. For any $x>0$, we have

$$
\mathbb{Q}\left(X_{\tau_{U}}-U>x \mid X_{\tau_{U}}-U>0, J_{\tau_{U}}=j\right)=\mathrm{e}^{-\eta_{j 1} x} .
$$

Similarly, for downward jumps, we have

$$
\mathbb{Q}\left(X_{\tau_{L}}-L<-x \mid X_{\tau_{L}}-L<0, J_{\tau_{L}}=j\right)=\mathrm{e}^{-\eta_{j 2} x},
$$

where $\tau_{L} \triangleq \inf \left\{t>0: X_{t} \leq L\right\}, L<0$.

\section{A.2 Fluidization}

Observe that the path of the regime-switching jump-diffusion process $\left(X_{t}, J_{t}\right)_{t \geq 0}$ in $(5)$ exhibits discontinuities, due to the presence of the compound Poisson process $\left(N_{t}^{j}\right)_{t \geq 0}, j=$ $1, \ldots, d$ under each regime $j$. Following the ideas of Asmussen et al. (2004), we can remove all the jumps from the original process $\left(X_{t}, J_{t}\right)_{t \geq 0}$ through a transformation, called fluidization. We hereafter denote the transformed process as $\left(\tilde{X}_{t}, \tilde{J}_{t}\right)_{t \geq 0}$ and call it the fluid process.

In simple terms, the fluid model $\left(\tilde{X}_{t}, \tilde{J}_{t}\right)_{t \geq 0}$ is constructed by replacing an upward jump by a linear segment with slope of 1 and a downward jump by a linear segment with slope of -1 . To move from the original regime-switching jump-diffusion model to its fluid counterpart, we augment the following states. Denote by $E_{(j, 0)}, E_{(j,+)}$ and $E_{(j,-)}$ the states in which the process behaves as a pure diffusion, exhibits an upward jump and a downward jump, respectively, when the state is $j \in E$. Under such a characterization, the transformed process no longer possesses jump, whence it has continuous sample paths. The state space of the regime-switching fluid model is denoted by

$$
\tilde{E}=\left\{E_{(1,0)}, E_{(1,+)}, E_{(1,-)}, E_{(2,0)}, E_{(2,+)}, E_{(2,-)}, \ldots, E_{(d, 0)}, E_{(d,+)}, E_{(d,-)}\right\},
$$

and the process indicating the underlying state by $\left(\tilde{J}_{t}\right)_{t \geq 0}$.

Note that the time frame under the fluid model is different from that of the original model. To restrict the elongated time so that the stopping time under the fluid model has the same distribution as the stopping time under the original model, we follow the concepts adopted in Jiang and Pistorius (2008) to define the virtual time and its right-continuous inverse.

Definition A.3. A function $T: \mathbb{R} \rightarrow \mathbb{R}$ is called a virtual time and is defined as, for every $t \geq 0$,

$$
T(t)=\int_{0}^{t} 1_{\left\{\tilde{J}_{s} \in E_{0}\right\}} \mathrm{d} s,
$$

where $E_{0} \triangleq\left\{E_{(1,0)}, \ldots, E_{(d, 0)}\right\} \subset \tilde{E}$. The right-continuous inverse of $T$ is defined as

$$
T^{-1}(s)=\inf \{t \geq 0: T(t)>s\} .
$$


From Definition A.3, the virtual time $T(t)$ takes out all the elongated time due to jumps. Furthermore, by the definition of the inverse $T^{-1}(s)$ of the virtual time, it follows that $\left(\tilde{X}_{T^{-1}(t)}, \tilde{J}_{T^{-1}(t)}\right)$ and $\left(X_{t}, J_{t}\right)$ have the same distribution.

Note that the restriction also applies to stopping times, and thus one can conclude that $T\left(\tilde{\tau}_{B}\right)$ and $\tau_{B}$ agree almost surely, where $\tau_{B}$ is a stopping time of the original model and $\tilde{\tau}_{B}$ is the corresponding stopping time of the fluid model. By adopting the concept of virtual time $T(t)$ in Definition A.3, finding the first passage time distribution under the original model $\left(X_{t}, J_{t}\right)_{t \geq 0}$ is equivalent to finding the first passage time distribution under the fluid model $\left(\tilde{X}_{t}, \tilde{J}_{t}\right)_{t \geq 0}$ :

Lemma A.4. Let $T(t)$ be the virtual time of the fluid model. For a stopping time $\tau_{B}$ of the original jump model, we have $T\left(\tilde{\tau}_{B}\right)=\tau_{B}$ (pathwise) almost surely, where $\tilde{\tau}_{B}$ is the corresponding stopping time of the fluid model.

\section{A.3 Finding $\mathbb{E}_{i}\left[\mathrm{e}^{-a \tau_{B}+b X_{\tau_{B}}}\right]$}

With the concepts of conditional independence and memoryless properties and the fludization in place, we now return to our study on the first passage time distribution of the regimeswitching jump-diffusion process $\left(X_{t}, J_{t}\right)_{t \geq 0}$ first exiting a pre-specified interval $[L, U]$.

The definition of $\tau_{B}$ in (11) entails that it is a stopping time with respect to the $\sigma$-algebra $\sigma\left(X_{t}, J_{t}\right)$ generated by $\left(X_{t}, J_{t}\right)_{t \geq 0}$, i.e. for any $t \geq 0$, we have

$$
\left\{\tau_{B}<t\right\} \in \sigma\left(X_{t}, J_{t}\right)
$$

In addition, this first passage time problem also includes the single-barrier passage times, where one can obtain the solutions from the double-barrier problem immediately by taking $U \rightarrow \infty$ for the first passage time to the lower barrier and by $L \rightarrow-\infty$ for the first passage time to the upper barrier.

Using Lemma A.4, we have

$$
\mathbb{E}_{i}\left[\mathrm{e}^{-a \tau_{B}+b X_{\tau_{B}}}\right]=\mathbb{E}_{i}\left[\mathrm{e}^{-a T\left(\tilde{\tau}_{B}\right)+b \tilde{X}_{\tilde{\tau}_{B}}}\right], \text { for } \quad a>0, \quad b \in \mathbb{R} \backslash\left\{\eta_{j 1},-\eta_{j 2}, j=1, \ldots, d\right\},
$$

where $\tilde{\tau}_{B}$ is the first passage time of $\left(\tilde{X}_{t}, \tilde{J}_{t}\right)_{t \geq 0}$ defined by

$$
\tilde{\tau}_{B} \triangleq \inf \left\{t>0: \tilde{X}_{t} \notin[L, U]\right\}
$$

and $T(t)$ is the virtual time of the fluid model defined in Definition A.3. Hence, it suffices to solve

$$
\mathbb{E}_{i}\left[\exp \left(-a T\left(\tilde{\tau}_{B}\right)+b \tilde{X}_{\tilde{\tau}_{B}}\right)\right]
$$

As in the case of $\tau_{B}$, the definition of $\tilde{\tau}_{B}$ also implies that it is a stopping time with respect to the filtration $\sigma\left(\tilde{X}_{t}, \tilde{J}_{t}\right)$. In addition to the given restriction on $b$, we also assume that $b>0$ in order to study the joint distribution of $T\left(\tilde{\tau}_{B}\right)$ and $\tilde{X}_{\tilde{\tau}_{B}}$. 
The fluid process $\left(\tilde{X}_{t}, \tilde{J}_{t}\right)_{t \geq 0}$ has continuous sample paths, whence either $\tilde{X}_{\tilde{\tau}_{B}}=U$ or $\tilde{X}_{\tilde{\tau}_{B}}=L$ possibly occurs. Therefore, from (44), it is logical to define

$$
\pi_{(i, j)}^{(k, \Delta)}[a] \triangleq \mathbb{E}_{i}\left[\exp \left(-a T\left(\tilde{\tau}_{B}\right)\right) 1_{\left\{\tilde{\tau}_{\tilde{\tau}_{B}}=(j, k), \tilde{X}_{\tilde{\tau}_{B}}=\Delta\right\}}\right]
$$

where $i, j \in\{1, \ldots, d\}, k \in\{0,+,-\}$, and $\Delta \in\{L, U\}$. For example, the event $\left\{\tilde{J}_{\tilde{\tau}_{B}}=\right.$ $\left.(j,+), \tilde{X}_{\tilde{\tau}_{B}}=U\right\}$ corresponds to the case of an overshoot, while the event $\left\{\tilde{J}_{\tilde{\tau}_{B}}=(j, 0), \tilde{X}_{\tilde{\tau}_{B}}=\right.$ $U\}$ corresponds to the situation that the process diffuses to the upper barrier $U$ with no overshoot. The downward case can be interpreted analogously.

In terms of (45), define

$$
\boldsymbol{\pi}[a] \triangleq\left(\pi_{(i, 1)}^{(0, U)}[a], \pi_{(i, 1)}^{(+, U)}[a], \ldots, \pi_{(i, d)}^{(0, L)}[a], \pi_{(i, d)}^{(-, L)}[a]\right)^{\top} .
$$

In view of $(46)$, it is now clear that Theorem 2.7 provides the representation of $\mathbb{E}_{i}\left[\exp \left(-a T\left(\tilde{\tau}_{B}\right)\right)\right]$, which in turn provides the representation of $\mathbb{E}_{i}\left[\exp \left(-a \tau_{B}\right)\right]$ by Lemma A.4.

The proofs of Theorem 2.7 and Corollary 2.8 in Section 2.2 hinge on the following lemma.

Lemma A.5. Suppose that

$$
-\infty<-\eta_{d 2}<\ldots<-\eta_{12}<0<\eta_{11}<\ldots<\eta_{d 1}<\infty .
$$

Then, for any $a>0$, the equation

$$
\operatorname{det}\left(\tilde{\boldsymbol{K}}_{a}[u]\right)=0
$$

where $\tilde{\boldsymbol{K}}_{a}[u]$ is defined in (12), has a total of $4 d$ distinct real roots. Moreover, let $\varrho_{1, a}<\cdots<$ $\varrho_{4 d, a}$ be the roots. Then, these roots are located as follows

$$
\begin{aligned}
-\infty<\varrho_{1, a}<-\eta_{d 2} & <\varrho_{2, a}<-\eta_{(d-1) 2}<\ldots<-\eta_{12}<\varrho_{2 d-1, a}<\beta_{2, a}<\varrho_{2 d, a}<0 \\
<\varrho_{2 d+1, a} & <\beta_{1, a}<\varrho_{2 d+2, a}<\eta_{11}<\varrho_{d+3, a}<\ldots<\eta_{(d-1) 1}<\varrho_{4 d-1, a}<\eta_{d 1}<\varrho_{4 d, a}<\infty
\end{aligned}
$$

where $\beta_{1, a}$ and $\beta_{2, a}$ are the roots of $g_{1}(s) \triangleq \kappa_{1}(u)-a+q_{11}=0$ such that $-\eta_{12}<\beta_{2, a}<0<$ $\beta_{1, a}<\eta_{11}$.

\section{Proof. (Sketch of Proof)}

It suffices to consider the case of $d=2$, as the proof for the $d>2$ regimes follows analogously.

Consider the case when $d=2$. Direct computation shows that, for $d=2$,

$$
\operatorname{det}\left(\tilde{\mathbf{K}}_{a}[u]\right) \triangleq f(u)=g_{1}(u) g_{2}(u)-q_{11} q_{22}
$$

where $g_{j}(u)=\kappa_{j}(u)-a-q_{j j}$ with $\kappa_{j}(u)$, for $j=1,2$, takes the form in (4). Under the given assumption, we observe that

$$
g_{j}\left(\eta_{j 1}-\right)=g_{j}\left(-\eta_{j 2}+\right)=+\infty, \quad g_{j}\left(\eta_{j 1}+\right)=g_{j}\left(-\eta_{j 2}-\right)=-\infty,
$$

which immediately implies that

$$
f\left(\eta_{j 1}-\right)=f\left(-\eta_{j 2}+\right)=+\infty, \quad f\left(\eta_{j 1}+\right)=f\left(-\eta_{j 2}-\right)=-\infty .
$$


In addition, we also see that $f(+\infty)=+\infty$ and $f(-\infty)=+\infty$. Hence, since $f(u)$ is continuous except at the singularities $\eta_{11}, \eta_{21},-\eta_{12}$, and $-\eta_{22}$, there exists at least one root at each of the intervals, $\left(-\infty,-\eta_{22}\right),\left(-\eta_{22},-\eta_{12}\right),\left(\eta_{11}, \eta_{21}\right)$, and $\left(\eta_{21}, \infty\right)$.

To obtain the remaining roots, since $\beta_{1, a}$ and $\beta_{2, a}$ are the roots of $g_{1}(u)=0$, we have $f\left(\beta_{1, a}\right)=$ $f\left(-\beta_{2, a}\right)=-q_{11} q_{22}<0$. Furthermore, observe that $f(0)=\left(a+q_{11}\right)\left(a+q_{22}\right)-q_{11} q_{22}>0$. Thus, since $f(u)$ is continuous on the interval $\left(-\eta_{12}, \eta_{11}\right)$, there exists at least one root at each of the intervals, $\left(-\eta_{12}, \beta_{2, a}\right),\left(\beta_{2, a}, 0\right),\left(0, \beta_{1, a}\right)$, and $\left(\beta_{1, a}, \eta_{11}\right)$.

So far, we have found eight distinct real roots for $f(u)=0$. Since $f(u)$ is a polynomial of degree 8 , the proof is completed.

We are now ready to prove Theorem 2.7 and Corollary 2.8 .

\section{Proof of Theorem 2.7}

Let $Y_{t}=-a T(t) / b$ and $Z_{t}=\tilde{X}_{t}+Y_{t}$, and define the matrix-valued process

$$
\mathbf{M}(a, b, t) \triangleq \int_{0}^{t} \mathrm{e}^{b Z_{s}} \mathbf{1}_{\tilde{J}_{s}} \mathrm{~d} s \tilde{\mathbf{K}}_{0}[b]+\mathrm{e}^{b Z_{0}} \mathbf{1}_{\tilde{J}_{0}}-\mathrm{e}^{b Z_{t}} \mathbf{1}_{\tilde{J}_{t}}+b \int_{0}^{t} \mathrm{e}^{b Z_{s}} \mathbf{1}_{\tilde{J}_{s}} \mathrm{~d} Y_{s},
$$

where $\mathbf{1}_{\tilde{J}_{t} \in(j, k)}$, with $j \in\{1, \ldots, d\}, k \in\{0,+,-\}$, denotes a row vector of length of $\tilde{\mathbf{K}}_{a}$ with all zeros but a one on the position corresponding to regime $j$, phase $k$. The matrix $\tilde{\mathbf{K}}_{a}[b]$ is defined by (12). Since the sample paths of $\left(\tilde{X}_{t}, \tilde{J}_{t}\right)_{t \geq 0}$ are continuous, we can apply Theorem $2(\mathrm{~d})$ of Asmussen and Kella (2000) to conclude that $\mathbf{M}(a, t)$ is a zero-mean martingale. Note that

$$
b \int_{0}^{t} \mathrm{e}^{b Z_{s}} \mathbf{1}_{\tilde{J}_{s}} \mathrm{~d} Y_{s}=-a \int_{0}^{t} \mathrm{e}^{b Z_{s}} \mathbf{1}_{\tilde{J}_{s}} 1_{\left\{\tilde{J}_{s} \in E_{0}\right\}} \mathrm{d} s=-a \int_{0}^{t} \mathrm{e}^{b Z_{s}} \mathbf{1}_{\tilde{J}_{s}} \mathbb{I}^{+} \mathrm{d} s
$$

where $\mathbb{I}^{+}$is the $3 d \times 3 d$ diagonal matrix with 1 on positions $E_{(j, 0)}$ or 0 elsewhere. It follows that

$$
\begin{aligned}
\mathbf{M}(a, b, t) & =\int_{0}^{t} \exp \left(b \tilde{X}_{t}-a T(t)\right) \mathbf{1}_{\tilde{J}_{s}} \mathrm{~d} s\left(\tilde{\mathbf{K}}_{0}[b]-a \mathbb{I}^{+}\right)+\mathrm{e}^{b x} \mathbf{1}_{\tilde{J}_{0}}-\exp \left(b \tilde{X}_{t}-a T(t)\right) \mathbf{1}_{\tilde{J}_{t}} \\
& =\int_{0}^{t} \exp \left(b \tilde{X}_{t}-a T(t)\right) \mathbf{1}_{\tilde{J}_{s}} \mathrm{~d} s \tilde{\mathbf{K}}_{a}[b]+\mathrm{e}^{b x} \mathbf{1}_{\tilde{J}_{0}}-\exp \left(b \tilde{X}_{t}-a T(t)\right) \mathbf{1}_{\tilde{J}_{t}} .
\end{aligned}
$$

In particular, post-multiplying the zero eigenvector $\mathbf{h}^{l}[a]$ of $\tilde{\mathbf{K}}_{a}\left[\varrho_{l, a}\right]$ defined in (13) to $\mathbf{M}\left(a, \varrho_{l, a}, t\right)$, where $l=1, \ldots, 4 d$, we obtain the zero-mean vector-valued martingale

$$
\tilde{\mathbf{M}}\left(a, \varrho_{l, a}, t\right) \triangleq \mathrm{e}^{\varrho_{l, a} x} \mathbf{1}_{\tilde{J}_{0}} \mathbf{h}^{l}[a]-\exp \left(\varrho_{l, a} \tilde{X}_{t}-a T(t)\right) \mathbf{1}_{\tilde{J}_{t}} \mathbf{h}^{l}[a],
$$

which, together with Doob's optional sampling theorem $\mathbb{E}\left[\tilde{\mathbf{M}}\left(a, \varrho_{l, a}, t \wedge \tilde{\tau}_{B}\right)\right]=0$, yields that

$$
\mathrm{e}^{\varrho l, a}{ }^{\ell} \mathbf{1}_{\tilde{J}_{0}} \mathbf{h}^{l}[a]=\mathbb{E}\left[\exp \left(\varrho_{l, a} \tilde{X}_{\tilde{\tau}_{B}}-a T\left(\tilde{\tau}_{B}\right)\right) \mathbf{1}_{\tilde{J}_{\tilde{\tau}_{B}}}\right] \mathbf{h}^{l}[a], \quad l=1, \ldots, 4 d .
$$

The terms $\mathbb{E}\left[\exp \left(\varrho_{l, a} \tilde{X}_{\tilde{\tau}_{B}}-a T\left(\tilde{\tau}_{B}\right)\right) \mathbf{1}_{\tilde{J}_{\tilde{\tau}_{B}}}\right]$ can be readily computed by observing that

$$
\tilde{X}_{\tilde{\tau}_{B}}=\left\{\begin{array}{lll}
L & \text { for } & \tilde{J}_{\tilde{\tau}_{B}}=(j, 0),(j,-), \\
U & \text { for } & \tilde{J}_{\tilde{\tau}_{B}}=(j, 0),(j,+)
\end{array}\right.
$$


The system of linear equations in (17) now follows by noting that $\tilde{J}_{0}=(i, 0)$ and decomposing the expectation in (48) with respect to the $\pi[a]$ 's defined in (46).

Parallel to the first passage time distribution under the regime-switching Brownian motion framework in Guo (2001), the existence of the solution to the system of linear equations in (17) is established by noting that (17) is known as the Vandermonde system of linear equations. The Vandermonde system of linear equations is uniquely solvable if and only if the associated function in (47) has $4 d$ distinct roots (see Lemma A.5).

\section{Proof of Corollary 2.8}

First note that

$$
1_{\left\{J_{\tau_{B}}=j\right\}}=1_{\left\{\tilde{J}_{T\left(\tilde{\tau}_{B}\right)}=(j, 0)\right\}}+1_{\left\{\tilde{J}_{T\left(\tilde{\tau}_{B}\right)}=(j,+)\right\}}+1_{\left\{\tilde{J}_{T\left(\tilde{\tau}_{B}\right)}=(j,-)\right\}} \cdot
$$

Recall that the event $\left\{\tilde{J}_{T\left(\tilde{\tau}_{B}\right)}=(j, 0)\right\}$ corresponds to the situation that the process $\left(\tilde{X}_{t}, \tilde{J}_{t}\right)_{t \geq 0}$ diffuses to either the upper barrier $U$ or the lower barrier $L$ when $J_{\tau_{B}}=j$, but not to result in an overshoot or undershoot. The Laplace transforms of $X_{\tau_{B}}$ for these cases are simply given by

$$
\hat{f}_{(j, 0)}^{U}(U) \triangleq \mathrm{e}^{b U}, \quad \hat{f}_{(j, 0)}^{L}(L) \triangleq \mathrm{e}^{b L},
$$

respectively. It follows that

$$
\begin{aligned}
\mathbb{E}_{i}\left[\mathrm{e}^{-a \tau_{B}+b X_{\tau_{B}}} 1_{\left\{\tilde{J}_{T\left(\tilde{\tau}_{B}\right)}=(j, 0)\right\}}\right] & =\mathbb{E}_{i}\left[\mathrm{e}^{-a \tau_{B}+b U} 1_{\left\{\tilde{\tilde{\tau}}_{\tilde{\tau}_{B}}=(j, 0), \tilde{X}_{\tilde{\tau}_{B}}=U\right\}}\right]+\mathbb{E}_{i}\left[\mathrm{e}^{-a \tau_{B}+b L} 1_{\left\{\tilde{\tilde{\tau}}_{\tilde{\tau}_{B}}=(j, 0), \tilde{X}_{\tilde{\tau}_{B}}=L\right\}}\right] \\
& =\pi_{(i, j)}^{(0, U)} \mathrm{e}^{b U}+\pi_{(i, j)}^{(0, L)} \mathrm{e}^{b L}
\end{aligned}
$$

where the $\pi[a]$ 's are given by (45).

Next, the event $\left\{\tilde{J}_{T\left(\tilde{\tau}_{B}\right)}=(j,+)\right\}$ corresponds to the case of an overshoot, when $J_{\tau_{B}}=j$. From Corollary A.2, we know that the overshoot $X_{\tau_{B}}-U$ is independent of $\tau_{B}$ and exponentially distributed. Hence, we obtain

$$
\begin{aligned}
\mathbb{E}_{i}\left[\mathrm{e}^{-a \tau_{B}+b X_{\tau_{B}}} 1_{\left\{\tilde{J}_{T\left(\tilde{\tau}_{B}\right)}=(j,+)\right\}}\right] & =\mathrm{e}^{b U} \mathbb{E}_{i}\left[\mathrm{e}^{-a \tau_{B}+b\left(X_{\tau_{B}}-U\right)} 1_{\left\{\tilde{\tilde{\tau}}_{\tilde{\tau}_{B}}=(j,+)\right\}}\right] \\
& =\mathrm{e}^{b U} \mathbb{E}_{i}\left[\mathrm{e}^{-a \tau_{B}} 1_{\left\{\tilde{J}_{\tilde{\tau}_{B}}=(j,+)\right\}}\right] \mathbb{E}_{i}\left[\mathrm{e}^{b\left(X_{\tau_{B}}-U\right)} 1_{\left\{\tilde{J}_{\tilde{\tau}_{B}}=(j,+)\right\}}\right] \\
& =\pi_{(i, j)}^{(+, U)} \hat{f}_{(j,+)}^{U}(U),
\end{aligned}
$$

where

$$
\hat{f}_{(j,+)}^{U}(U) \triangleq \mathrm{e}^{b U} \int_{0}^{\infty} \mathrm{e}^{b y} \eta_{j 1} \mathrm{e}^{-\eta_{j 1} y} \mathrm{~d} y=\frac{\eta_{j 1}}{\eta_{j 1}-b} \mathrm{e}^{b U} .
$$

Similarly, for the case of an undershoot, we have

$$
\mathbb{E}_{i}\left[\exp \left(-a \tau_{B}+b X_{\tau_{B}}\right) 1_{\left\{\tilde{J}_{T\left(\tilde{\tau}_{B}\right)}=(j,+)\right\}}\right]=\pi_{(i, j)}^{(-, L)} \hat{f}_{(j,-)}^{L}(L)
$$

where

$$
\hat{f}_{(j,-)}^{L}(L) \triangleq \mathrm{e}^{b L} \int_{-\infty}^{0} \mathrm{e}^{b y} \eta_{j 2} \mathrm{e}^{\eta_{j 2} y} \mathrm{~d} y=\frac{\eta_{j 2}}{\eta_{j 2}+b} \mathrm{e}^{b L} .
$$

Summing the terms $\pi_{(i, j)}^{(k, b)} \hat{f}_{(j, k)}^{\Delta}(b)$, where $\hat{f}_{(j, k)}^{\Delta}(b)$ is defined in $(19)$, for $i, j \in\{1, . ., d\}, k \in$ $\{0,+,-\}$, and $\Delta \in\{L, U\}$, the desired result follows. 


\section{B Appendix: Valuation of fractional lookback option}

Consider a fractional floating strike lookback put option with the equity-indexed benefit function at the exponentially-distributed maturity $T_{\mathrm{x}}$ with mean $\frac{1}{\alpha}$ (see $(21)$ ) given by

$$
b_{f \text { Lookback }}\left(T_{\mathrm{x}}, S_{T_{\mathrm{x}}}\right) \triangleq\left(\gamma \max _{0 \leq t \leq T_{\mathrm{x}}} S_{t}-S_{T_{\mathrm{x}}}\right)^{+}
$$

where $\gamma \in(0,1]$. We valuate this fractional floating strike lookback put option at time 0 , i.e.

$$
\mathbb{E}\left[\mathrm{e}^{-r T_{\mathrm{x}}}\left(\gamma \max _{0 \leq t \leq T_{\mathrm{x}}} S_{t}-S_{T_{\mathrm{x}}}\right)^{+}\right]=\mathbb{E}\left[\mathrm{e}^{-r T_{\mathrm{x}}} S_{T_{\mathrm{x}}}\left(\gamma \max _{0 \leq t \leq T_{\mathrm{x}}} \frac{S_{t}}{S_{T_{\mathrm{x}}}}-1\right)^{+}\right] .
$$

Define $\tilde{\mathbb{Q}}$ as an equivalent martingale measure via the Radon-Nikodym derivative

$$
\left.\frac{\mathrm{d} \tilde{\mathbb{Q}}}{\mathrm{d} \mathbb{Q}}\right|_{\mathcal{F}_{T_{\mathrm{x}}}}=\mathrm{e}^{-r T_{\mathrm{x}}} \frac{S_{T_{\mathrm{x}}}}{S_{0}}
$$

under which $S_{T_{\mathrm{x}}}$ is the numéraire. Then, it follows that

$$
S_{0} \mathbb{E}\left[\mathrm{e}^{-r T_{\mathrm{x}}} \frac{S_{T_{\mathrm{x}}}}{S_{0}}\left(\gamma \max _{0 \leq t \leq T_{\mathrm{x}}} \frac{S_{t}}{S_{T_{\mathrm{x}}}}-1\right)^{+}\right]=S_{0} \tilde{\mathbb{E}}\left[\left(\gamma \max _{0 \leq t \leq T_{\mathrm{x}}} \mathrm{e}^{X_{t}-X_{T_{\mathrm{x}}}}-1\right)^{+}\right]
$$

Hence, we can apply Proposition 2.6 to obtain the dynamics of $\max _{0 \leq t \leq T_{\mathrm{x}}} \mathrm{e}^{X_{t}-X_{T_{\mathrm{x}}}}$ under measure $\tilde{\mathbb{Q}}$, provided that we know the dynamics of $\max _{0 \leq t \leq T_{\mathrm{x}}} \mathrm{e}^{X_{t}-X_{T_{\mathrm{x}}}}$ under $\mathbb{Q}$. To compute the distribution of $\max _{0 \leq t \leq T_{\mathrm{x}}} \mathrm{e}^{X_{t}-X_{T_{\mathrm{x}}}}$ under $\mathbb{Q}$, we first need to know the distribution of $\mathrm{e}^{X_{t}-X_{T_{\mathrm{x}}}}$ under $\mathbb{Q}$, for all $t \in\left[0, T_{\mathrm{x}}\right]$. For the case of Lévy processes, the answer is immediate. The property of stationary increments of Lévy processes immediately implies that

$$
\mathrm{e}^{X_{t}-X_{T_{\mathrm{x}}}} \stackrel{D}{=} \mathrm{e}^{X_{t-T_{\mathrm{x}}}} \stackrel{D}{=} \mathrm{e}^{-X_{T_{\mathrm{x}}-t}}
$$

Unfortunately, the property of stationary increments generally does not hold in our regimeswitching model, due to the presence of the Markov chain. For the special case when the Markov chain $\left(J_{t}\right)_{t \geq 0}$ is reversible, the property of stationary increments is maintained and we can still compute the distribution of $\mathrm{e}^{X_{t}-X_{T_{\mathrm{x}}}}$. In the remaining section, we complete the analysis on the fractional lookback option under the additional assumption that the Markov chain $\left(J_{t}\right)_{t \geq 0}$ is reversible.

Lemma B.1. Let $\left(X_{t}, J_{t}\right)_{t \geq 0}$ be the regime-switching, double-exponential jump-diffusion process in Definition 2.1. If the Markov chain $\left(J_{t}\right)_{t \geq 0}$ is also reversible, then

$$
\max _{0 \leq t \leq T_{\mathrm{x}}} \mathrm{e}^{X_{t}-X_{T_{\mathrm{x}}}} \stackrel{D}{=} \max _{0 \leq t \leq T_{\mathrm{x}}} \mathrm{e}^{-X_{T_{\mathrm{x}}-t}}
$$

Proof. For $t \geq 0$, let $\left\{t_{0}<\ldots<t_{N}\right\}$ be an arbitrary partition on $\left[t, T_{\mathrm{x}}\right]$, i.e. $t=t_{0}<\ldots<t_{N}=$ $T_{\mathrm{x}}$. Let $u_{0}, u_{1}, \ldots, u_{N} \in \mathbb{R}$. We first study the joint distribution of $\left(X_{t_{0}}-X_{t_{N}}, \ldots, X_{t_{N-1}}-X_{t_{N}}\right)$. 
Since $u_{k}\left[X_{t_{k}}-X_{t_{N}}\right]=u_{k}\left[\sum_{l=k+1}^{N}\left(X_{t_{l-1}}-X_{t_{l}}\right)\right]$, it follows that

$$
\begin{aligned}
& u_{0}\left[\sum_{l=1}^{N}\left(X_{t_{l-1}}-X_{t_{l}}\right)\right]+u_{1}\left[\sum_{l=2}^{N}\left(X_{t_{l-1}}-X_{t_{l}}\right)\right]+\cdots+u_{N-2}\left[\sum_{l=N-1}^{N}\left(X_{t_{l-1}}-X_{t_{l}}\right)\right] \\
& +u_{N-1}\left[X_{t_{N-1}}-X_{t_{N}}\right] \\
= & u_{0}\left[X_{t_{0}}-X_{t_{1}}\right]+\left(u_{0}+u_{1}\right)\left[X_{t_{1}}-X_{t_{2}}\right]+\cdots+\left(\sum_{l=1}^{N-1} u_{l-1}\right)\left[X_{t_{N-2}}-X_{t_{N-1}}\right] \\
& +\left(\sum_{l=1}^{N} u_{l-1}\right)\left[X_{t_{N-1}}-X_{t_{N}}\right] .
\end{aligned}
$$

Using (51), the joint moment generating function of $\left(X_{t_{0}}-X_{t_{N}}, \ldots, X_{t_{N-1}}-X_{t_{N}}\right)$ becomes

$$
\begin{aligned}
& \mathbb{E}_{J_{t_{0}}}\left\{\exp \left(u_{0}\left[X_{t_{0}}-X_{t_{N}}\right]+u_{1}\left[X_{t_{1}}-X_{t_{N}}\right]+\cdots+u_{N-2}\left[X_{t_{N-2}}-X_{t_{N}}\right]+u_{N-1}\left[X_{t_{N-1}}-X_{t_{N}}\right]\right)\right\} \\
= & \mathbb{E}_{J_{t_{0}}}\left\{\exp \left(u_{0}\left[X_{t_{0}}-X_{t_{1}}\right]+\left(u_{0}+u_{1}\right)\left[X_{t_{1}}-X_{t_{2}}\right]+\cdots+\left(\sum_{l=1}^{N-1} u_{l-1}\right)\left[X_{t_{N-2}}-X_{t_{N-1}}\right]\right)\right. \\
& \left.\cdot \mathbb{E}_{J_{t_{N-1}}}\left\{\exp \left[\left(\sum_{l=1}^{N} u_{l-1}\right)\left(X_{t_{N-1}}-X_{t_{N}}\right)\right]\right\}\right\} \\
= & \left(\mathbf{C}_{1}\right)_{J_{t_{0}} J_{t_{1}}} \cdot\left(\mathbf{C}_{2}\right)_{J_{t_{1}} J_{t_{2}}} \cdots\left(\mathbf{C}_{N}\right)_{J_{t_{N-1}} J_{t_{N}}},
\end{aligned}
$$

where we define $\mathbf{C}_{m} \triangleq \exp \left[\left(\left\{\kappa_{k}\left(-\sum_{l=1}^{m} u_{l-1}\right)\right\}_{\text {diag }}+\mathbf{Q}\right)\left(t_{m}-t_{m-1}\right)\right]$, for $m=1, \ldots, N$.

In the case when the Markov chain $\left(J_{t}\right)_{t \geq 0}$ is reversible, $\mathbf{Q}$ is then symmetric and therefore matrices $\mathbf{C}_{m}$, for $m=1, \ldots, N$, are commutative. Therefore, the joint moment generating function of $\left(X_{t_{0}}-X_{t_{N}}, \ldots, X_{t_{N-1}}-X_{t_{N}}\right)$ in (52) equals to

$$
\begin{aligned}
\left(\mathbf{C}_{1}\right)_{J_{t_{0} J_{t_{1}}}} \cdot\left(\mathbf{C}_{2}\right)_{J_{t_{1} J_{t_{2}}}} \cdots\left(\mathbf{C}_{N}\right)_{J_{t_{N-1} J_{t_{N}}}}=\left[\left(\mathbf{C}_{1}\right)_{J_{t_{0}} J_{t_{1}}} \cdot\left(\mathbf{C}_{2}\right)_{J_{t_{1}} J_{t_{2}}} \cdots\left(\mathbf{C}_{N}\right)_{J_{t_{N-1}} J_{t_{N}}}\right]^{\top} \\
=\left(\mathbf{C}_{N}\right)_{J_{t_{N}} J_{t_{N-1}}} \cdots\left(\mathbf{C}_{2}\right)_{J_{t_{2}} J_{t_{1}}} \cdot\left(\mathbf{C}_{1}\right)_{J_{t_{1}} J_{t_{0}}} \cdot(53)
\end{aligned}
$$

On the other hand, the joint moment generating function of $\left(-X_{t_{N}-t_{0}}, \ldots,-X_{t_{N}-t_{N-1}}\right)$ is

$$
\begin{aligned}
& \mathbb{E}_{J_{t_{N}}}\left\{\exp \left[-u_{N-1} X_{t_{N}-t_{N-1}}-u_{N-2} X_{t_{N}-t_{N-2}}-\cdots-u_{0} X_{t_{N}-t_{0}}\right]\right\} \\
= & \mathbb{E}_{J_{t_{N}}}\left\{\operatorname { e x p } \left[-\left(\sum_{l=1}^{N} u_{l-1}\right)\left(X_{t_{N}-t_{N-1}}\right)-\left(\sum_{l=1}^{N-1} u_{l-1}\right)\left(X_{t_{N}-t_{N-2}}-X_{t_{N}-t_{N-1}}\right)\right.\right. \\
& \left.\left.-\cdots-\left(u_{0}+u_{1}\right)\left(X_{t_{N}-t_{1}}-X_{t_{N}-t_{2}}\right)\right] \cdot \mathbb{E}_{J_{t_{N}-t_{1}}}\left\{\exp \left[-u_{0}\left(X_{t_{N}-t_{0}}-X_{t_{N}-t_{1}}\right)\right]\right\}\right\} \\
= & \left(\mathbf{C}_{N}\right)_{J_{t_{N}-t_{N}} J_{t_{N}-t_{N-1}}} \cdots\left(\mathbf{C}_{1}\right)_{J_{t_{N}-t_{1} J_{t_{N}-t_{0}}}}
\end{aligned}
$$


where last equality follows by observing that

$$
\begin{aligned}
& \mathbb{E}_{J_{t_{N}-t_{m}}}\left\{\exp \left[-\left(\sum_{l=1}^{m} u_{l-1}\right)\left(X_{t_{N}-t_{m-1}}-X_{t_{N}-t_{m}}\right)\right]\right\} \\
= & \left(\exp \left[\left(\left\{\kappa_{k}\left(-\sum_{l=1}^{m} u_{l-1}\right)\right\}_{\operatorname{diag}}+\mathbf{Q}\right)\left(\left(t_{N}-t_{m-1}\right)-\left(t_{N}-t_{m}\right)\right)\right]\right)_{J_{t_{N}-t_{m}} J_{t_{N}-t_{m-1}}} \\
= & \left(\mathbf{C}_{m}\right)_{J_{t_{N}-t_{m}} J_{t_{N}-t_{m-1}}} .
\end{aligned}
$$

By the assumption that Markov chain $\left(J_{t}\right)_{t \geq 0}$ is reversible, we have

$$
\left(J_{t_{N}-t_{0}}, J_{t_{N}-t_{1}}, \cdots, J_{t_{N}-t_{N-1}}\right) \stackrel{D}{=}\left(J_{t_{0}}, J_{t_{1}}, \cdots, J_{t_{N-1}}\right) .
$$

Combining (53), (54), and (55), it follows that

$$
\begin{aligned}
& \mathbb{E}_{J_{t_{0}}}\left\{\exp \left(u_{0}\left[X_{t_{0}}-X_{t_{N}}\right]+u_{1}\left[X_{t_{1}}-X_{t_{N}}\right]+\cdots+u_{N-2}\left[X_{t_{N-2}}-X_{t_{N}}\right]+u_{N-1}\left[X_{t_{N-1}}-X_{t_{N}}\right]\right)\right\} \\
= & \left(\mathbf{C}_{N}\right)_{J_{t_{N}} J_{t_{N-1}}} \cdots\left(\mathbf{C}_{2}\right)_{J_{t_{2}} J_{t_{1}}} \cdot\left(\mathbf{C}_{1}\right)_{J_{t_{1} J_{t_{0}}}}=\left(\mathbf{C}_{N}\right)_{J_{t_{N}-t_{N}} J_{t_{N}-t_{N-1}}} \cdots\left(\mathbf{C}_{1}\right)_{J_{t_{N}-t_{1}} J_{t_{N}-t_{0}}} \\
= & \mathbb{E}_{J_{t_{N}}}\left\{\exp \left[-u_{N-1} X_{t_{N}-t_{N-1}}-u_{N-2} X_{t_{N}-t_{N-2}}-\cdots-u_{0} X_{t_{N}-t_{0}}\right]\right\} .
\end{aligned}
$$

In other words, when Markov chain $\left(J_{t}\right)_{t \geq 0}$ is reversible, we can conclude that

$$
\left(X_{t_{0}}-X_{T_{\mathrm{x}}}, \ldots, X_{t_{N-1}}-X_{T_{\mathrm{x}}}\right) \stackrel{D}{=}\left(-X_{T_{\mathrm{x}}-t_{0}}, \ldots,-X_{T_{\mathrm{x}}-t_{N-1}}\right) .
$$

Since the partition $\left\{t_{0}<\ldots<t_{N}\right\}$ is chosen arbitrarily, we can apply Kolmogorov's Extension Theorem to show that

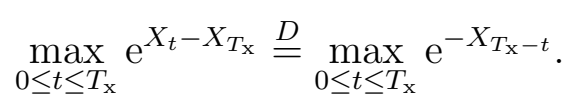

By Lemma B.1, the fractional floating-strike lookback put option in (50) becomes

$$
\begin{aligned}
S_{0} \mathbb{E}\left[\mathrm{e}^{-r T_{\mathrm{x}}} \frac{S_{T_{\mathrm{x}}}}{S_{0}}\left(\gamma \max _{0 \leq t \leq T_{\mathrm{x}}} \frac{S_{t}}{S_{T_{\mathrm{x}}}}-1\right)^{+}\right] & =S_{0} \tilde{\mathbb{E}}\left[\left(\gamma \max _{0 \leq t \leq T_{\mathrm{x}}} \mathrm{e}^{X_{t}-X_{T_{\mathrm{x}}}}-1\right)^{+}\right] \\
& =S_{0} \tilde{\mathbb{E}}\left[\left(\gamma \max _{0 \leq t \leq T_{\mathrm{x}}} \mathrm{e}^{-X_{T_{\mathrm{x}}-t}}-1\right)^{+}\right]
\end{aligned}
$$

indicating that the fair valuation the fractional floating-strike lookback option is essentially analogous to the fair valuation of the floating-strike lookback put option, albeit under measure $\tilde{\mathbb{Q}}$. 


\section{Appendix: Numerical Laplace inversion}

In this paper, we adopt the Fourier-series method first developed by Abate and Whitt (1992) on the positive real line and later extended to the entire real line by Petrella (2004), calling it the bilateral Abate-Whitt algorithm. The benefits of the Fourier-series method are that the methodology provides error bounds and converges rapidly. We summarize the results of Petrella (2004) in this appendix.

Denoted by $\widehat{F}(\alpha)$ the Laplace transform of the function $F(t)$ with respect to $t \in \mathbb{R} \backslash\{0\}$. Then $F(t)$ can be recovered from $\widehat{F}(\alpha)$ by the bilateral Abate-Whitt algorithm on $\mathbb{R}$ :

$$
F(t)=\frac{\mathrm{e}^{A / 2}}{2 t} \Re\left(\widehat{F}\left(\frac{A}{2 t}\right)\right)+\frac{\mathrm{e}^{A / 2}}{t} \sum_{j=1}^{\infty} \Re\left(\widehat{F}\left(\frac{A+2 j \pi \mathrm{i}}{2 t}\right)\right)+e_{d},
$$

where $\mathrm{i}=\sqrt{-1}, A$ is an arbitrary positive constant controlling the discretization error $e_{d}$, and $e_{d}=e_{d}^{+}+e_{d}^{-}$, with

$$
\left.\left.e_{d}^{+}=\sum_{j=1}^{\infty} \mathrm{e}^{-j A} F((2 j+1) t)\right) \quad \text { and } \quad e_{d}^{-}=\sum_{j=-\infty}^{-1} \mathrm{e}^{-j A} F((2 j+1) t)\right) \text {. }
$$

Observe that (57) is almost identical to the Fourier-series method proposed by Abate and Whitt (1992). The main difference here is that the function $F$ is defined on $\mathbb{R}$, whereas Abate-Whitt approximation scheme works on the functions defined on $\mathbb{R}_{+}$.

To assess the accuracy of (57), Abate and Whitt (1992) provide the discretization error bound for the case when $t>0$. Assuming that $F$ is bounded, i.e. $F(t)<C$ for some $C$, the discretization error $e_{d}=e_{d}^{+}$of the Abate-Whitt method can be bounded by

$$
e_{d}<C \frac{\mathrm{e}^{-A}}{1-\mathrm{e}^{-A}} \simeq C \mathrm{e}^{-A}
$$

Thus, we should set $A$ large enough to make the error small. However, because of roundoff errors, increasing $A$ would make inversion harder. In practice, Abate and Whitt (1992) suggest that the choice of $A=18$ should produce stable and accurate results. For the bilateral Abate-Whitt algorithm, Cai and Kou (2011) numerically show that $A \in[15,55]$ yields stable and accurate results.

For the case of (57), Theorem 1 of Petrella (2004) shows that we still maintain exponential error bounds:

Theorem C.1. Suppose that $t_{0} \in\left(A / \theta^{-}, A / 2 \theta^{+}\right)$and

$$
F\left((2 j+1) t_{0}\right) \leq \begin{cases}C^{+}\left(\theta^{+}\right) \mathrm{e}^{2 \theta^{+} j t_{0}}, \theta^{+}>0, & \text { for } j \geq 0, \\ C^{-}\left(\theta^{-}\right) \mathrm{e}^{\theta^{-} j t_{0}}, \theta^{-}>0 & \text { for } j<0,\end{cases}
$$

with $\theta^{-}>2 \theta^{+}>0$ and $C^{+}\left(\theta^{+}\right), C^{-}\left(\theta^{-}\right)$positive continuous functions, we can then obtain the following exponential bounds for both discretization errors in (57):

$$
e_{d}^{+}=C^{+}\left(\theta^{+}\right) \frac{\mathrm{e}^{-\left(A-2 \theta^{+} t_{0}\right)}}{1-\mathrm{e}^{-\left(A-2 \theta^{+} t_{0}\right)}} \quad \text { and } \quad e_{d}^{-}=C^{-}\left(\theta^{-}\right) \frac{\mathrm{e}^{-\left(\theta^{-} t_{0}-A\right)}}{1-\mathrm{e}^{-\left(\theta^{-} t_{0}-A\right)}} .
$$


As remarked in Petrella (2004), the exponential bounds in Theorem C.1 only exist for the values of the inversion point on a specific interval. In practice, however, this is not a significant constraint, as we can often choose an appropriate inversion point by rescaling the inverted function $F($.$) . Note that for the case when the Laplace transform is performed on \mathbb{R}_{+}$, as in the case of Abate and Whitt (1992), no rescaling parameter is necessary and one can simply follow (57) to invert the Laplace transform on $t>0$.

Observe that (57) is an infinite-series representation. To obtain a high degree of accuracy, we need to add a large number of terms. However, a large number of summands would certainly hinder the speed of inversion. Fortunately, close inspection of the Abate-Whitt algorithm reveals that it is in terms of an alternating series, which can be well approximated by an appropriate binomial expansion. To speed up the inversion procedure, we can modify $F(t)$ by using the Euler algorithm

$$
F(t) \approx \sum_{k=0}^{m}\left(\begin{array}{c}
m \\
k
\end{array}\right) 2^{-m} S_{n+k}(t)
$$

where

$$
S_{n}(t)=\frac{\mathrm{e}^{A / 2}}{2 t} \Re\left(\widehat{F}\left(\frac{A}{2 t}\right)\right)+\frac{\mathrm{e}^{A / 2}}{t} \sum_{k=1}^{n} \Re\left(\widehat{F}\left(\frac{A+2 k \pi \mathrm{i}}{2 t}\right)\right) .
$$

By employing the Euler algorithm, we find that any $n>40$ and $m>15$ produces stable results. Since the summation involves less than 100 terms, the algorithm is very efficient.

\section{References}

[1] Abate, J., Whitt, W. (1992). The Fourier-series method for inverting transforms of probability distributions. Queueing Systems, 10 (1-2) 5-88.

[2] Albizzati , M.-O., and Gemen, H. (1994). Interest rate risk management and valuation of the surrender option in life insurance policies. The Journal of Risk and Insurance, 61(4) 616-637.

[3] Asmussen, S. (2003). Applied Probability and Queues. Springer-Verlag, New York.

[4] Asmussen, S., Avram, F., Pistorius, M. (2004) Russian and American put options under exponential phase-type Lévy models. Stochastic Processes and their Applications, 109(1) 79-111.

[5] Asmussen, S., Kella, O. (2000) A multi-dimensional martingale for Markov additive processes and its applications. Advances in Applied Probability, 32 376-393.

[6] Bacinello, A. R. (2001). Fair pricing of life insurance participating contracts with a minimum interest rate guarantees. ASTIN Bulletin, 31(2) 275-297.

[7] Black, F., Scholes, M. (1973). The pricing of options and corporate liabilities. Journal of Political Economy, 81(3) 637-654. 
[8] Botta, R. F., Harris, C. M. (1986). Approximation with generalized hyperexponential distributions: Weak convergence results. Queueing Systems, 2 169-190.

[9] Boyle, P., Draviam, T. (2007). Pricing exotic options under regime switching. Insurance: Mathematics and Economics, 40 (2) 267-282.

[10] Boyle, P., Schwartz, E. (1977). Equilibrium prices of guarantees under equity-linked contracts. The Journal of Risk and Insurance, 44(4) 639-680.

[11] Bowers, N., Gerber, H. U., Hickman, J., Jones, D., Nesbitt, C. (1997). Actuarial Mathematics. Second Ed., Society of Actuary, Schaumburg, Ill.

[12] Brennan, M., Schwartz, E. (1976). The pricing of equity-linked life insurance policies with an asset value guarantee. Journal of Financial Economics, 3(3) 195-213.

[13] Brennan, M., Schwartz, E. (1979). Alternative investment strategies for the issuers of equity linked life insurance policies with an asset value guarantee. Journal of Business, 52(1) 63-93.

[14] Cai, N., Kou, S. (2011). Option pricing under a mixed-exponential jump diffusion model. Management Science, 57(11) 2067-2081.

[15] Chan, B. (1990). Ruin probability for translated combination of exponential claims. ASTIN Bulletin, 20(1) 113-114.

[16] Cont, R., Tankov, P. (2004). Financial Modelling with Jump Processes. Springer-Verlag, New York.

[17] Dong, Y. (2011). Fair valuation of life insurance contracts under a correlated jump diffusion model. ASTIN Bulletin, 41(2) 429-447

[18] Dufresne, D. (2007). Fitting combinations of exponentials to probability distributions. Applied Stochastic Models in Business and Industry, 23 23-48.

[19] Dufresne, D., Gerber, H. (1988). The probability and severity of ruin for combinations of exponential claim amount distributions and their translations. Insurance: Mathematics and Economics, 7(2) 75-80.

[20] Dufresne, D., Gerber, H. (1991). Risk theory for the compound Poisson process that is perturbed by diffusion. Insurance: Mathematics and Economics, 10(1) 51-59.

[21] Elliott, R.J., Aggoun, L., Moore, J. (1994). Hidden Markov Models: Estimation and Control. Springer-Verlag, Berlin-Heidelberg-New York.

[22] Elliott, R. J., Siu, T. K. (2009). On Markov-modulated exponential-affine bond price formulae. Applied Mathematical Finance, 16(1) 1-15.

[23] Geman, H., Albizzati, M.-O. (1994). Interest Rate Risk Management and Valuation of the Surrender Option in Life Insurance Policies. The Journal of Risk and Insurance, 61(4) 616-637.

[24] Gerber, H. U., Shiu, E. S. W. (2006). Maximizing dividends without bankruptcy. ASTIN Bulletin, 36(1) 5-23. 
[25] Gerber, H. U., Shiu, E. S. W., and Yang, H. (2012). Valuing equity-linked death benefits and other contingent options: A discounted density approach. Insurance: Mathematics and Economics, (51) 73-92.

[26] Gerber, H. U., Shiu, E. S. W., and Yang, H. (2013). Valuing equity-linked death benefits in jump diffusion models. Insurance: Mathematics and Economics, (53) 615-623.

[27] Guo, X. (2001). An explicit solution to an optimal stopping problem with regime switching. Journal of Applied Probability, 38(2) 464-481.

[28] Hamilton, J. D. (2008) A new approach to the economic analysis of non-stationary time series and the business cycle. Econometrica, 57 (2) 357-384.

[29] Hardy, M. R. (2001) A regime-switching model of long-term stock returns. North American Actuarial Journal, 5 (2) 41-53.

[30] Jiang, Z., Pistorius, M. (2008) On perpetual American put valuation and first passage in a regime-switching model with jumps. Finance and Stochastics, 12 331-355.

[31] Ko, B., Shiu, E.S.W, Wei, L. (2010). Pricing maturity guarantee with dynamic withdrawal benefit. Insurance: Mathematics and Economics, 47(2) 216-223.

[32] Kou, S. G. (2002). A jump-diffusion model for option pricing. Management Science, 48 1086-1101.

[33] Kou, S. G., Wang, H. (2003) First passage times for a jump-diffusion process. Advances in Applied Probability, 48 504-531.

[34] Lee, H. (2003). Pricing equity-indexed annuities with path-dependent options. Insurance: Mathematics and Economics, (33) 677-690.

[35] Lin, X. S., Liu, X. (2007). Markov aging process and phase-type law of mortality. North American Actuarial Journal, 11(4) 92-109.

[36] Merton, R. C. (1976). Option pricing when underlying stock returns are discontinuous. Journal of Financial Economics 3(1) 125-144.

[37] Mijatović, A., and Pistorius, M. (2011). Exotic derivatives in a dense class of stochastic volatility models with jumps. Advanced Mathematical Methods for Finance. Springer, $455-508$.

[38] Milevsky, M. A., Posner, S. E. (2001). The titanic option: valuation of the guaranteed minimum death benefit in a variable annuities and mutual funds. The Journal of Risk and Insurance, 68(1) 93-128.

[39] Petrella, G. (2004). An extension of the Euler Laplace transform algorithm with applications in option pricing. Operations Research Letters, 32 380-389.

[40] Ronn, E. I., Verma, A. K. (2004). Pricing risk-adjusted deposit insurance: An optionbased model. Journal of Finance, 41(4) 871-895.

[41] Schweizer, M. (2010). Minimal Entropy Martingale Measure. Encyclopedia of Quantitative Finance, Cont, R. (ed.) Wiley, 1195-1200. 
[42] Siu, C. C. (2012). Financial Modeling with Markov Additive Processes. Ph.D. Thesis Tokyo Metropolitan University.

[43] Siu, T. K. (2005). Fair valuation of participating policies with surrender options and regime switching. Insurance: Mathematics and Economics, 37(3) 871-895.

[44] Ulm, E. R. (2006). The effect of the real option to transfer on the value of guaranteed minimum death benefits. The Journal of Risk and Insurance, 73(1) 533-552.

[45] Ulm, E. R. (2008). Analytical solution for return of premium and rollup guaranteed minimum death benefit options under some simple mortality laws. ASTIN Bulletin, 38(2) 543-563.

[46] Yuen. F. L., Yang, H. (2009). Option pricing in a jump-diffusion model with regime switching. ASTIN Bulletin, 39(2) 515-539. 